\title{
Distinct Heterosynaptic Plasticity in Fast Spiking and Non-Fast-Spiking Inhibitory Neurons in Rat Visual Cortex
}

\author{
Marina Chistiakova, ${ }^{1 \star}$ Vladimir Ilin, ${ }^{1 \star}$ Matvey Roshchin, ${ }^{2}{ }^{\oplus}$ Nicholas Bannon, ${ }^{1}{ }^{\circledR}$ Alexey Malyshev, ${ }^{2}$ \\ ¿Zoltán Kisvárday, ${ }^{3}$ and Maxim Volgushev ${ }^{1,2}$ \\ ${ }^{1}$ Department for Psychological Sciences, University of Connecticut, Storrs, Connecticut 06269, 2 Institute of Higher Nervous Activity and Neurophysiology of \\ Russian Academy of Sciences, Moscow, 117485, Russia, and ${ }^{3}$ MTA-DE Neuroscience Research Group, Department of Anatomy, Histology and Embryology, \\ University of Debrecen, 4032 Debrecen, Hungary
}

Inhibition in neuronal networks of the neocortex serves a multitude of functions, such as balancing excitation and structuring neuronal activity in space and time. Plasticity of inhibition is mediated by changes at both inhibitory synapses, as well as excitatory synapses on inhibitory neurons. Using slices from visual cortex of young male rats, we describe a novel form of plasticity of excitatory synapses on inhibitory neurons, weight-dependent heterosynaptic plasticity. Recordings from connected pyramid-to-interneuron pairs confirm that postsynaptic activity alone can induce long-term changes at synapses that were not presynaptically active during the induction, i.e., heterosynaptic plasticity. Moreover, heterosynaptic changes can accompany homosynaptic plasticity induced in inhibitory neurons by conventional spike-timing-dependent plasticity protocols. In both fast-spiking (FS) and non-FS neurons, heterosynaptic changes were weightdependent, because they correlated with initial paired-pulse ratio (PPR), indicative of initial strength of a synapse. Synapses with initially high PPR, indicative of low release probability ("weak" synapses), had the tendency to be potentiated, while synapses with low initial PPR ("strong" synapses) tended to depress or did not change. Interestingly, the net outcome of heterosynaptic changes was different in FS and non-FS neurons. FS neurons expressed balanced changes, with gross average $(n=142)$ not different from control. Non-FS neurons $(n=66)$ exhibited net potentiation. This difference could be because of higher initial PPR in the non-FS neurons. We propose that weight-dependent heterosynaptic plasticity may counteract runaway dynamics of excitatory inputs imposed by Hebbian-type learning rules and contribute to fine-tuning of distinct aspects of inhibitory function mediated by FS and non-FS neurons in neocortical networks.

Key words: excitatory synapses; heterosynaptic plasticity; inhibitory neurons; plasticity; visual cortex

Significance Statement

Dynamic balance of excitation and inhibition is fundamental for operation of neuronal networks. Fine-tuning of such balance requires synaptic plasticity. Knowledge about diverse forms of plasticity operating in excitatory and inhibitory neurons is necessary for understanding normal function and causes of dysfunction of the nervous system. Here we show that excitatory inputs to major archetypal classes of neocortical inhibitory neurons, fast-spiking (FS) and non-fast-spiking (non-FS), express a novel type of plasticity, weightdependent heterosynaptic plasticity, which accompanies the induction of Hebbian-type changes. This novel form of plasticity may counteract runaway dynamics at excitatory synapses to inhibitory neurons imposed by Hebbian-type learning rules and contribute to fine-tuning of diverse aspects of inhibitory function mediated by FS and non-FS neurons in neocortical networks.

\section{Introduction}

Inhibitory neurons with profoundly diverse morphology, distinct electrophysiology, and pattern of protein expression, serve a multitude of functions in neocortical networks. Ultimate classification of inhibitory neurons should be thus functional, determined by the combination of their inputs and, most importantly,

\footnotetext{
Received Dec. 2, 2018; revised June 19, 2019; accepted June 21, 2019.

Author contributions: M.C. and M.V. designed research;V.I., M.R., A.M., Z.K., and M.V. performed research; M.C., V.I., M.R., N.B., A.M., Z.K., and M.V. analyzed data; N.B., Z.K., and M.V. contributed unpublished reagents/analytic tools; M.C., V.I., and M.V. wrote the paper.

This work was supported by National Institutes of Health Grant R01 MH087631 to M.V., and by the Russian Foundation for Basic Research Grants 18-015-00397 to A.M., and NAP-2-2017-1.2.1-NKP-2017-00002 to Z.K. We thank Stanislav Volgushev for his help with statistical analysis.

The authors declare no competing financial interests.
}

*M.C. and V.I. contributed equally to this work.

Correspondence should be addressed to Maxim Volgushev at maxim.volgushev@uconn.edu.

V. Ilin's present address: Pittsburgh Institute for Neurodegenerative Diseases and Department of Neurology, University of Pittsburgh School of Medicine, Pittsburgh, Pennsylvania 15213.

N. Bannon's present address: Department of Neurobiology, Weinberg School of Arts and Sciences, Northwestern University, Evanston, Illinois 60208.

https://doi.org/10.1523/JNEUROSCI.3039-18.2019

Copyright $\odot 2019$ the authors 
axonal projections. Classes defined solely by morphology, electrophysiology, or protein expression have partial overlap, but do not coincide completely with the functional classification (Kawaguchi and Kubota, 1997; Markram et al., 2004; Ascoli et al., 2008; Battaglia et al., 2013; DeFelipe et al., 2013; Jiang et al., 2015; Tremblay et al., 2016). From an electrophysiological perspective, the majority of inhibitory neurons in the neocortex can be attributed to one of the two archetypal classes: fast spiking (FS) and non-fast spiking (non-FS; Druckmann et al., 2013). Most FS neurons are basket or chandelier cells, and express parvalbumin (PV) but not somatostatin (SST). Non-FS neurons exhibit diverse spiking patterns, including adapting, bursting, low-threshold spiking (LTS), and irregular; belong to diverse morphological types, e.g., Martinotti, bipolar, bitufted, and non-basket multipolar; express SST and other marker-proteins, but not PV (Kawaguchi and Kubota, 1997; Markram et al., 2004; Ascoli et al., 2008; Gentet, 2012; Battaglia et al., 2013; Druckmann et al., 2013; Jiang et al., 2015; Liguz-Lecznar et al., 2016; Tremblay et al., 2016).

Roles served by inhibition in cortical networks include maintenance of an overall excitation/inhibition balance (Wehr and 2003; Okun and Lampl, 2008; Ozeki et al., 2009; Moore et al., 2018), control over the spread of activity (Adesnik and Scanziani, 2010; Cardin, 2018), and shaping response selectivity (Vidyasagar et al., 1996; Monier et al., 2003; Barnes et al., 2015). Moreover, inhibition is a factor controlling cortical plasticity including critical periods (Hensch et al., 1998; Hensch, 2005; Froemke et al., 2007; Gandhi et al., 2008; Feldman, 2009; Dorrn et al., 2010; Liguz-Lecznar et al., 2016). To achieve these functions during development (Maffei et al., 2004, 2006; Garkun and Maffei, 2014; Miska et al., 2018) and in adult brain (Huang et al., 2013; Chen et al., 2015) inhibition is fine-tuned by synaptic plasticity. Plasticity of inhibition may be mediated by changes of inhibitory connections per se, as well as by plasticity of excitatory inputs to inhibitory neurons. Here we consider that latter locus.

Sparse studies of plasticity of excitatory inputs to inhibitory neurons in the neocortex report heterogeneous results. In slices from mouse visual or somatosensory cortex, long-term potentiation (LTP) was observed at excitatory inputs to FS neurons, but not in non-FS neurons (Sarihi et al., 2008). Another study found LTP in FS neurons only in presence of $\alpha 1$ and $\beta$ adrenergic agonists, and regular spike-timing-dependent plasticity (STDP) that required activation of NMDA and adrenergic receptors in SSTexpressing neurons (Huang et al., 2013). Strong theta-burst stimulation induced NMDA-independent LTP in SST-neurons (Chen et al., 2009). In slices from rat neocortex, LTS neurons expressed STDP, whereas FS neurons expressed only long-term depression (LTD) and no LTP before P17 (Lu et al., 2007) and LTP after P22 (Lefort et al., 2013). Moreover, during development, connections from L4 star pyramids to FS neurons can be potentiated by visual deprivation (Maffei et al., 2006).

These studies show that at least some excitatory synapses on inhibitory neurons express associative Hebbian-type plasticity. Hebbian-type rules introduce positive feedback on synaptic changes, making synapses prone to runaway potentiation or depression. However, synaptic weights in real neurons do not express runaway dynamics, but instead remain in an operational range. This implies the existence of additional mechanism(s) counteracting the runaway dynamics. Our prior experimental and computational analysis of plasticity in pyramidal neurons from visual and auditory cortex demonstrated that this role can be served by weight-dependent heterosynaptic plasticity; changes at synapses that were not active during the induction of plasticity (Volgushev et al., 2000; Lee et al., 2012; Chen et al., 2013; Volgu- shev et al., 2016; for review, see Chistiakova et al., 2014, 2015). Here we ask, whether excitatory synapses to inhibitory neurons of major electrophysiological classes express a similar form of heterosynaptic plasticity.

\section{Materials and Methods}

All experimental procedures are in compliance with the U.S. National Institutes of Health regulations and were approved by the Institutional Animal Care and Use Committee of the University of Connecticut.

\section{Preparation of slices}

Details of slice preparation and recording are similar to those used in previous studies (Volgushev et al., 2000; Lee et al., 2012; Bannon et al., 2014). Male Wistar rats (15-34 d old, Charles River Laboratories or Harlan) were anesthetized with isoflurane, decapitated, and the brain quickly removed and placed into an ice-cold oxygenated artificial CSF solution (ACSF), containing the following (in $\mathrm{mm}$ ): $125 \mathrm{NaCl}, 25$ $\mathrm{NaHCO}_{3}, 25$ glucose, $3 \mathrm{KCl}, 1.25 \mathrm{NaH}_{2} \mathrm{PO}_{4}, 2 \mathrm{CaCl}_{2}, 1 \mathrm{MgCl}_{2}$, bubbled with $95 \% \mathrm{O}_{2} / 5 \% \mathrm{CO}_{2}, \mathrm{pH}$ 7.4. Coronal slices ( $350 \mu \mathrm{m}$ thickness) containing the visual cortex were prepared from the right hemisphere. After at least $1 \mathrm{~h}$ recovery at room temperature, individual slices were transferred to a recording chamber mounted on an Olympus BX-50WI or Olympus BX-51WI microscope equipped with IR-DIC optics. For pair recording slices were incubated for $45 \mathrm{~min}$ at $34^{\circ} \mathrm{C}$ immediately after cutting and then kept at room temperature. During recordings slices were perfused with oxygenated ACSF at $28-32^{\circ} \mathrm{C}$.

\section{Intracellular recording}

Whole-cell recordings were made from inhibitory interneurons from layers $1,2 / 3,4$, and 5 of the visual cortex. Intracellular pipette solution contained, in mM: $130 \mathrm{~K}$-gluconate, $20 \mathrm{KCl}, 10 \mathrm{HEPES}, 10 \mathrm{Na}$ phosphocreatine, $4 \mathrm{Mg}$-ATP, $0.3 \mathrm{Na}_{2}$-GTP, pH 7.4 with $\mathrm{KOH}$. For recording, neurons of nonpyramidal shape were preselected. Neurons were classified electrophysiologically using responses to long depolarizing pulses $(0.2-1 \mathrm{~s})$, voltage-current relationships and parameters of their action potentials (APs). Some neurons were labeled with Neurobiotin (Vector Laboratories; $0.2 \%$ added to the intracellular solution) for morphological identification. For recordings from connected pairs of neurons, after patching a nonpyramidal neuron, several nearby pyramidal neurons were tested for a synaptic connection in a loose-patch configuration with the electrode filled with ACSF. After a connected neuron was found, it was patched with an electrode containing regular intracellular solution. Both presynaptic and postsynaptic neurons in recorded pairs were characterized electrophysiologically.

\section{Experimental design: synaptic stimulation}

Synaptic responses were evoked either by extracellular stimulation or by presynaptic spikes in paired recordings. In paired recordings, presynaptic APs were induced by brief $(5 \mathrm{~ms})$ depolarizing pulses applied in pairs (50 ms interval) each $10 \mathrm{~s}$. Evoked EPSCs were recorded at a holding potential between -70 and $-80 \mathrm{mV}$. In the main series of experiments extracellular stimulation was used. Monosynaptic EPSPs of small amplitude were evoked using two pairs of bipolar tungsten electrodes (S1 and S2) placed in the vicinity of the recorded neuron. Paired stimuli with a 50 ms interpulse interval were applied to $S 1$ and $S 2$ in alternating sequence, so that each input was stimulated with paired pulses each $15 \mathrm{~s}$. To verify that recorded postsynaptic responses are excitatory, several responses were recorded at depolarized potentials between -50 and $-40 \mathrm{mV}$, which is above the reversal potential for $\mathrm{Cl}^{-}$with our extracellular and intracellular solutions (Zhang et al., 2015). Only those PSPs that were still depolarizing (PSCs remained inward in paired recordings) at this membrane potential were considered excitatory and included in the analysis. To further confirm that recorded synaptic responses are excitatory and that observed plastic changes of these responses are not contaminated by changes of inhibition, a series of experiments was performed in the presence of the $\mathrm{GABA}_{\mathrm{A}}$ blocker picrotoxin $(50 \mu \mathrm{M}$; Sigma-Aldrich, catalog \#528105; CAS 124-87-8) in the recording solution. Membrane potential (or holding current in voltage-clamp mode used in paired recordings) and input resistance were monitored throughout experiments; cells in 
which either parameter changed by $>15 \%$ by the end of recording were discarded.

\section{Experimental design: plasticity induction}

Synaptic plasticity was induced by either a pairing (STDP) procedure or intracellular tetanization. Pairing procedure consisted of 30 bursts of 5 postsynaptic APs induced by brief depolarizing pulses $(5 \mathrm{~ms}, 5$ pulses at $100 \mathrm{~Hz}$ ), with one of the inputs (S1 or S2) stimulated $10 \mathrm{~ms}$ before each burst of spikes. The other input ( $\mathrm{S} 2$ or S1) was not stimulated during the pairing. Intracellular tetanization consisted of the same pattern of postsynaptic activation: 30 bursts of 5 APs induced by depolarizing pulses ( 5 ms, 5 pulses at $100 \mathrm{~Hz}$ ) through the recording electrode, but without any concurrent presynaptic stimulation. In recordings from connected pairs of neurons, intracellular tetanization was applied to the postsynaptic (inhibitory) neuron in bridge mode. Following the induction, test synaptic stimulation was resumed, and EPSP/EPSC responses were recorded for another $40-60 \mathrm{~min}$.

There are two broad types of synaptic changes: homosynaptic plasticity (at synapses activated during the induction) and heterosynaptic plasticity (at non-activated synapses). We use these definitions following the original paper on heterosynaptic plasticity by Lynch et al. (1977). With the induction protocols used in the present study, the term homosynaptic refers to plastic changes at synapses stimulated during the pairing procedure. Heterosynaptic are changes at synapses which were unpaired (not stimulated) during the pairing procedure. Also heterosynaptic are changes at any test inputs occurring after intracellular tetanization, because it is a purely postsynaptic protocol and no synapses were activated during the intracellular tetanization.

\section{Data processing and statistical analysis}

Data analysis was made using custom-written programs in MATLAB (MathWorks), and scripts in R (The R Foundation for Statistical Computing). All inputs included in the analysis fulfilled the following criteria: (1) excitatory nature of EPSP/EPSC, as verified by the absence of reversal when recorded at potentials between -40 and $-50 \mathrm{mV}$; (2) stability of EPSP/EPSC amplitudes during the control period; (3) stability of the membrane potential and input resistance throughout the recording; and (4) stability of the onset latency and kinetics of the rising slope of the EPSP/EPSC. Amplitudes of EPSPs (or EPSCs) are measured as the difference between the mean membrane potential (or current) during two time windows, the first time window placed before the onset and the second window placed on the rising slope of the synaptic response, just before the peak. Amplitude of the response to the second pulse in the paired-pulse stimulation paradigm was measured using windows of the same duration and separation, but shifted by the length of the interpulse interval (50 ms). The paired-pulse ratio (PPR) was calculated as the amplitude of the second EPSP (EPSC) divided by the amplitude of the first. For calculating significance of response amplitude changes after a plasticity-induction protocol at individual inputs, amplitudes of responses to the first pulse were used. $t$ Test was used to compare control responses recorded before the application of plasticity-induction protocol $(n=15-35$ responses from a stationary period just before plasticity induction) to responses after plasticity induction $(n=40-120$ responses from a period typically $\sim 30-60 \mathrm{~min}$ after the induction). Response changes (LTP or LTD) were considered significant at $p<0.05$. For calculation of population averages across inputs, response amplitudes in each input were first normalized to control, and then averaged across inputs. For correlations, Pearson's $r$ was used. $P$ values for Pearson correlations and $\chi^{2}$ tests were calculated using an online calculator (Social Science Statistics, 2018; https://www.socscistatistics.com/). In the text, $p$ values $>0.001$ are given in full and $p$ values $<0.001$ as $p<0.001$. In the figures significance is denoted as corresponding to $p$ values of ${ }^{\star} 0.05,{ }^{* *} 0.01$, and ${ }^{* *} 0.001$.

Identification of cell types: morphology and neuron reconstruction Reconstruction and morphological identification of cells recorded with Neurobiotin (Vector Laboratories; $0.2 \%$ added to the pipette solution) was done as following. For Neurobiotin injection into the cells current pulses applied during pairing procedure or intracellular tetanization (as in Experimental design: plasticity induction above) were sufficient; in some cases after plasticity experiment was completed, we applied an additional staining protocol consisting of anodal $0.05-0.2 \mathrm{nA}$ current pulses at $0.5 \mathrm{~s}$ duty cycles for 5-20 min. After withdrawing the recording pipette the injected cells were allowed to survive for 5-20 min. At the end of experiments slices were immediately transferred in fixative containing $4 \%$ paraformaldehyde and $1 \%$ glutaraldehyde in $0.1 \mathrm{~m}$ phosphate buffer, $\mathrm{pH} \mathrm{7.4}$, and stored in fixative at $4^{\circ} \mathrm{C}$ for $4-10 \mathrm{~d}$, until histological processing.

Histology. For visualizing labeled cells, slices were resectioned on a vibratome (Leica 1000S, Leica Biosystems) to $80 \mu \mathrm{m}$ thickness. To ensure the parallel alignment of slices with the sectioning plane of the vibratome, first a tissue block of 2-3 mm thickness (a piece of any fixed brain) was glued to the cutting stage and trimmed for providing a smooth surface parallel to the blade trajectory. Then the brain slice containing the region-of-interest was glued on the smooth tissue surface. For fostering flatness, the slice was gently pressed by touching it with a glass slide from above. Because the recorded cells were typically located 15-50 $\mu \mathrm{m}$ below the surface care was taken for gluing the slices with their opposite surface and resection them to $80 \mu \mathrm{m}$ thick sections. A total of $4-7$ sections per slice could be obtained of which the lowermost section was usually lost because of infiltration of the glue.

Sections of each slice were collected in a glass vial. For revealing neurobiotin label, the following histological protocol was used. Sections were washed for $10 \mathrm{~min}$ in $0.1 \mathrm{M} \mathrm{PB}$ and $10 \mathrm{~min}$ in $0.05 \mathrm{M}$ Tris buffered saline (TBS; pH 7.6) at room temperature. Then TBS containing $0.1 \%$ Triton $\mathrm{X}-100$ was used $2 \times 10 \mathrm{~min}$ followed by avidin-biotin-complexed-HRP (ABC-Elite, Vector Laboratories) diluted at 1:200 in $0.05 \mathrm{M}$ TBS containing $0.1 \%$ Triton $\mathrm{X}-100$ overnight at $4^{\circ} \mathrm{C}$. Next, the sections were washed in $0.05 \mathrm{~m}$ TBS $(2 \times 10 \mathrm{~min})$ and $0.05 \mathrm{~m}$ Tris-buffer (TB; pH 7.6, $10 \mathrm{~min})$ and incubated in $0.05 \%$ of 3,3-diaminobenzidine (Sigma-Aldrich) in TB containing $0.0025-0.005 \% \mathrm{CoCl}_{2}$ for $30 \mathrm{~min}$. The chromogen was visualized by adding $\mathrm{H}_{2} \mathrm{O}_{2}(0.01 \%$ at final concentration) to the incubating medium. After 1-3 min, the reaction was stopped by diluting out the reagent solution with an excess of TB in succession. Sections were postfixated in $\mathrm{OsO}_{4}$ (1\% in $0.1 \mathrm{M} \mathrm{PB}, 45 \mathrm{~min}$; Sigma-Aldrich) and dehydrated in ascending series of ethanol $(10 \mathrm{~min}$ each $)$ and $2 \times 10 \mathrm{~min}$ propylene oxide (Merck). Finally, the sections were transferred in resin (Durcupan ACM, Sigma-Aldrich) for overnight, mounted on slides, coverslipped and cured at $56^{\circ} \mathrm{C}$ in oven for $24 \mathrm{~h}$ (Freund et al., 1989).

Neuron reconstruction. Labeled neurons were reconstructed in 3-dimensions using the Neurolucida reconstruction system (Neurolucida v.5.65) and a Leica DMBR light microscope fitted with a computer controlled xyz-moving stage (Märzhäuser Wetzlar). For reconstructing cell bodies, dendrites, axons and axon terminals of each labeled neuron, two to four consecutive sections were used. Alignment of adjoining sections was made with the help of least-square fitting algorithm for corresponding cut ends of the labeled structures such as axonal and dendritic segments (Buzás et al., 1998). Cortical layers were defined based on the following morpho-histological features: layer 1 is a cell-sparse zone below which is a layer containing pyramidal cells (layer 2/3), with the size of pyramidal cells progressively increasing toward the bottom of layer 3; layer 4 contains a high density of small neurons; layer 5 displays the largest pyramidal cells; and layer 6 being often the thickest layer, which contains a neuronal population with variable morphology. The type of labeled neurons was determined using physiological and morphological criteria in line with the scheme of Ascoli et al. (2008). Figure 1, $A$ and $B$, shows examples of reconstructed Martinotti and basket cells and their electrophysiology.

\section{Identification of cell types: electrophysiological classification}

For electrophysiological classification of neurons, we used a combination of formal classification algorithms and expert classification. For measuring parameters of an AP, we first detected its threshold using two approaches. In the first "acceleration" approach used in our prior work (Naundorf et al., 2006; Baranauskas et al., 2010), the threshold was defined as the point at which $\mathrm{dV} / \mathrm{dt}$ reached $20 \mathrm{mV} / \mathrm{ms}$. In the second "geometrical" approach, the threshold was defined as the point on the AP trace that had maximal distance from the line connecting AP peak and a point on the trace $5 \mathrm{~ms}$ before the AP peak. For the formal classification 
we measured the following parameters of APs and firing patterns. For APs: voltage at the threshold, at the peak, and at the peak of afterhyperpolarization (AHP); amplitude (peak threshold); depth of AHP (threshold - AHP peak); peak-to-AHP voltage (peak - AHP peak); and ratio of AP amplitude to AHP amplitude (amplitude/depth of AHP). Using the timing of the threshold, AP peak, and AHP peak, we calculated: time to peak (timing of the peak - timing of the threshold), time from AP peak to AHP peak; and total AP time (timing of AHP peak - timing of the threshold). We also calculated the width of AP at 50\% amplitude and at $20 \%$ amplitude (Fig. 1). From responses to depolarizing current steps we calculated AP amplitude accommodation (ratio of the amplitudes of the last AP in the response to the amplitude of the first $\mathrm{AP}$; and ratio of the amplitudes of the second/first APs), interspike interval accommodation (the ratio of the last/ first interspike intervals, and the ratio of the interval between second and third AP divided by the interval between first and second APs); average, $\mathrm{SD}$, and coefficient of variation $(\mathrm{SD} /$ mean) of interspike intervals.

For the formal classification, we used a linear discriminant model ( $R$ version 3.4.0, functions $l d a$ and predict; The R Foundation for Statistical Computing, 2017). We first trained the model on a subset of neurons $(n=74)$ consisting of typical representatives of FS, non-FS, and pyramidal neurons. After training, we run multivariate classification on all neurons ( $n=$ 200 ). Results of the formal classification were then compared with the results of expert classification. After trying different combinations of the above parameters for classification of neurons, we have selected the following four: ratio of AP amplitude to AHP amplitude, AP width at $20 \%$ amplitude, time from the AP peak to the AHP peak, and coefficient of variation of interspike intervals (SD/mean). Selection of these parameters is in agreement with prior work on formal electrophysiological classification of cortical neurons (Battaglia et al., 2013; Druckmann et al., 2013).

Code accessibility. Custom-written software codes used for data processing are available upon request (maxim.volgushev@uconn.edu).

\section{Results}

\section{Classification of FS versus non-FS inhibitory neurons}

The multitude of functions served by inhibition in neocortical networks is mediated by the great variety of inhibitory neurons. Functional role of each class of inhibitory neurons is ultimately determined by a combination of their morphology including synaptic inputs and axonal projections, electrophysiology and protein expression. Classes of inhibitory neurons defined by subsets of these features commonly show an overlap, but do not coincide completely (Kawaguchi and Kubota, 1997; Markram et al., 2004; Ascoli et al., 2008; Battaglia et al., 2013; Jiang et al., 2015; Tremblay et al., 2016). Here we classify inhibitory neurons using electrophysiology and laminar location of the somata, complemented in some cases with reconstruction of the dendritic and axonal field and hence identification of the morphological type.
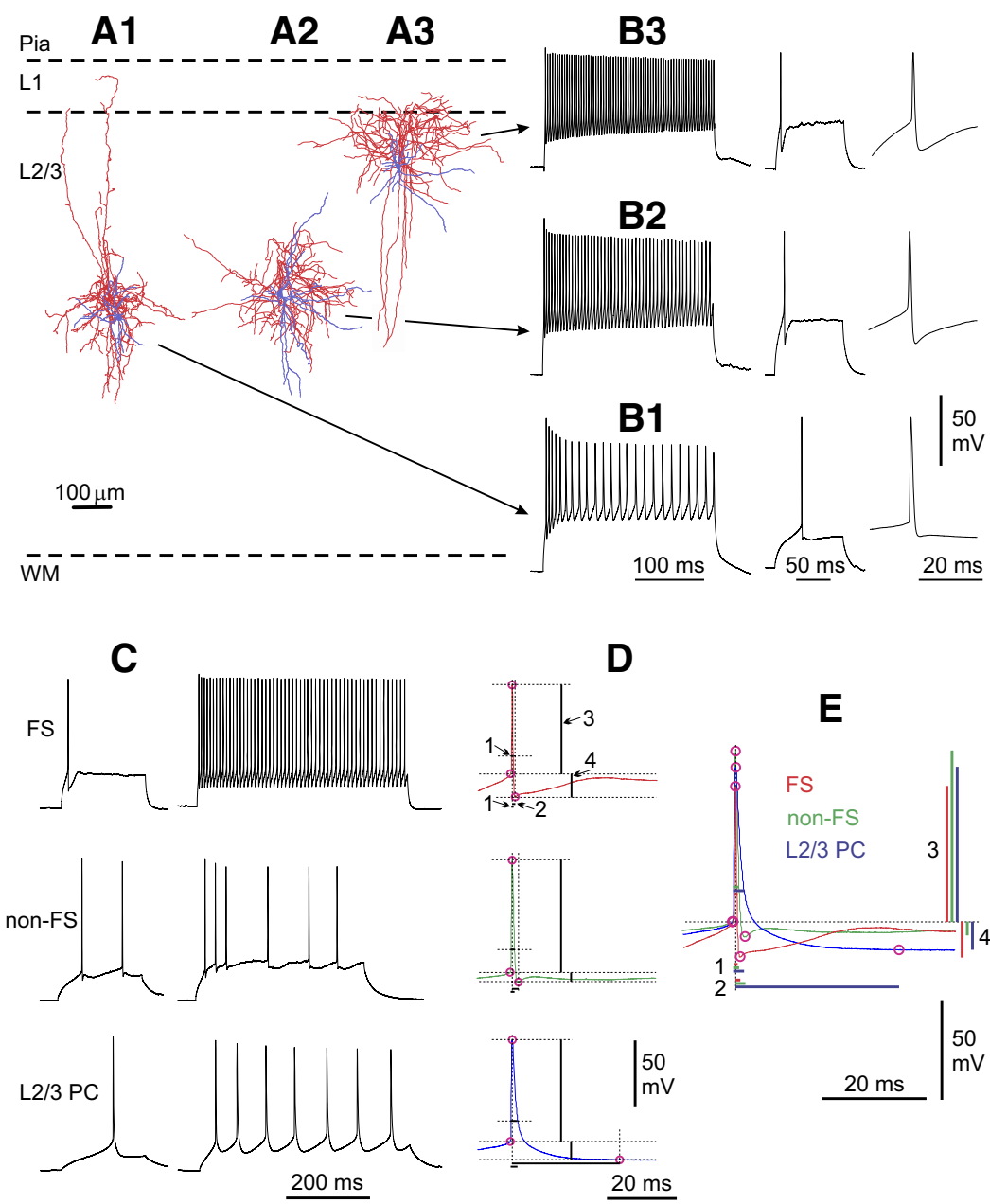

\begin{tabular}{l|l} 
& 50 \\
$20 \mathrm{~ms}$ & $\mathrm{mV}$
\end{tabular}
$20 \mathrm{~ms}$
Figure 1. Distinct morphology, firing patterns, and AP shapes in FS, non-FS interneurons, and $L 2 / 3$ pyramidal neurons in rat visual cortex. $A, B$, Distinct morphology and electrophysiology of FS and non-FS cells. A Martinotti cell from layer 4 with non-FS electrophysiology $(\boldsymbol{A 1}, \boldsymbol{B} 1)$, and two basket cells with FS electrophysiology, a large basket from layer $4(\boldsymbol{A 2}, \boldsymbol{B} 2)$, and a basket cell

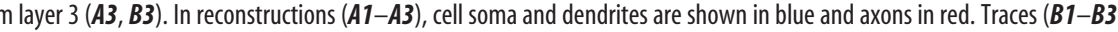

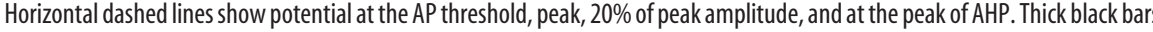
dicate the magnitude of measured parameters: AP width at 20\% amplitude (1), time from AP peak and AHP peak (2), AP amplitude from the threshold (3), and amplitude of AHP from the threshold (4). $\boldsymbol{E}$, Comparison of APs in FS, non-FS and L2/3 pyramidal neurons, with parameters 1-4 color-coded for FS, non-FS, and pyramidal cells.

From electrophysiological perspective, majority of inhibitory neurons (Battaglia et al., 2013; Druckmann et al., 2013) can be attributed to one of the two archetypal classes: FS and non-FS neurons. Figure 1 illustrates a typical non-FS neuron, a Martinotti cell from layer 4 (Fig. $1 A 1, B 1$ ), and two typical FS neurons, basket cells from layer 4 and layer 3 (Fig. $1 A 2, B 2$ and $A 3, B 3$ ). FS and non-FS neurons differ by the firing pattern in response to depolarizing current steps and by the waveform of the AP (Fig. $1 B 1$ vs B2,B3). Both classes of interneurons, FS and non-FS, differ from the spikes and firing patterns of pyramidal neurons (Fig. $1 C-E$ ).

For attributing the recorded neurons to electrophysiological classes of FS or non-FS cells we used a combination of expert classification and a formal classification algorithm. For formal electrophysiological classification we used the following AP parameters (Fig. $1 D, E$ ): width at $20 \%$ amplitude, time from the AP peak to the peak of AHP, ratio of AP peak amplitude measured 

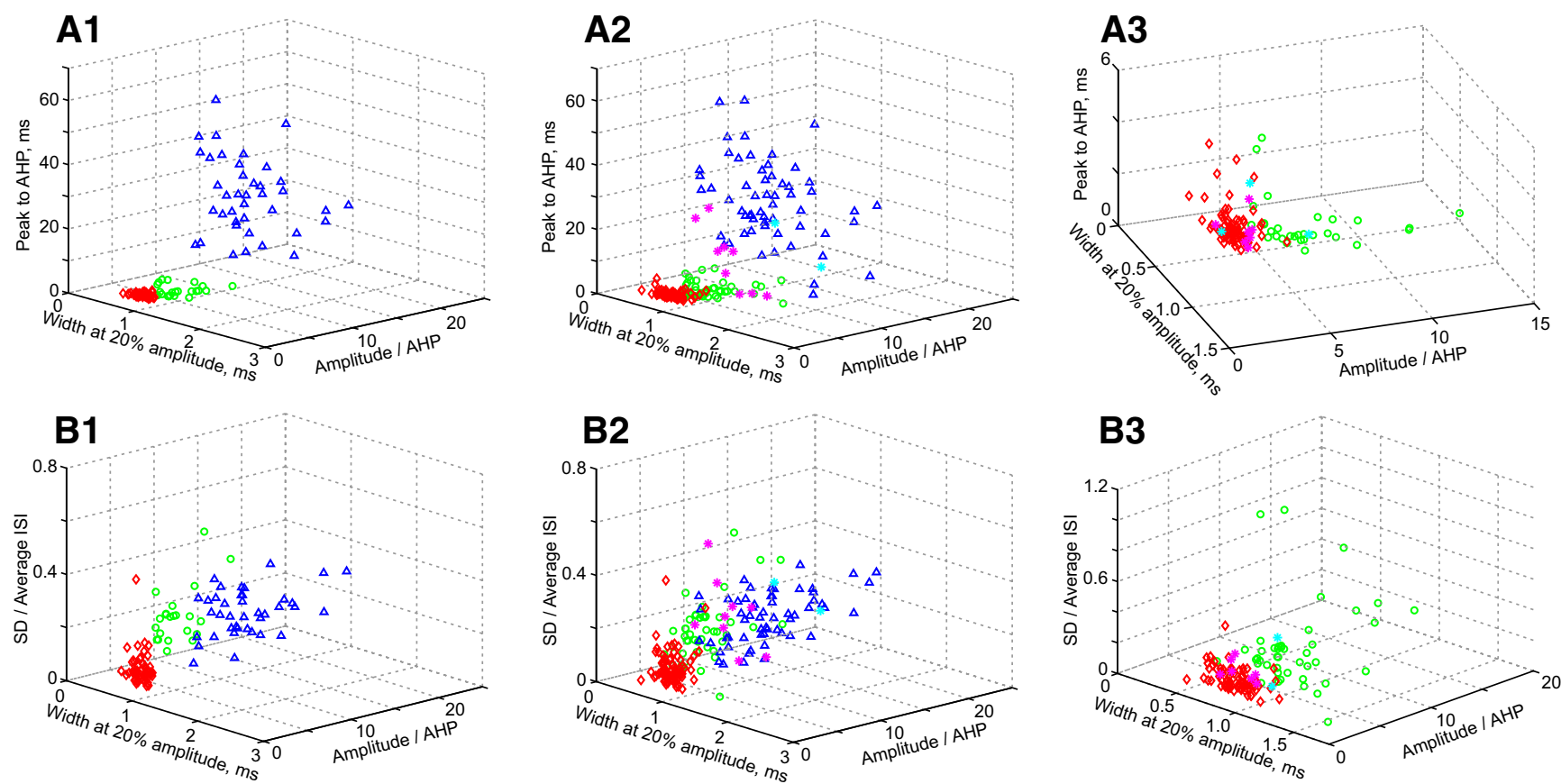

Figure 2. Formal classification of $F S$, non-FS, and pyramidal neurons. In scatter plots each point represents one neuron, with parameters of spikes as $X-Y-Z$ coordinates. A1-A3, Coordinate axes are as follows: AP width at $20 \%$ of amplitude, ratio of AP amplitude from threshold to the amplitude of AHP from threshold, and time from AP peak to AHP peak. B1-B3, Coordinate axes are as follows: AP width at $20 \%$ of amplitude, ratio of AP amplitude from threshold to the amplitude of AHP from threshold, and coefficient of variation, i.e., SD/average of interspike intervals (ISIs) in response to a long depolarizing pulse. $\boldsymbol{A 1}, \boldsymbol{B 1}$, Data for a subset of typical FS ( $n=53$; red, diamonds), non- $\mathrm{FS}$ ( $n=22$; green, circles), and pyramidal ( $n=39$; blue, triangles) neurons, which were used for training the classification algorithm. Note the clear segregation of pyramidal neurons from inhibitory neurons (FS and non-FS) in $\boldsymbol{A}$, and segregation of FS versus non-FS cells in B1. A2, B2, Classification of FS ( $n=80)$ and non-FS $(n=51)$ cells against pyramidal $(n=69)$ neurons. Magenta asterisks show cases that were expert-classified as pyramidal neurons, but attributed to the group of inhibitory neurons ( $F S$ and non-FS) by the formal classification algorithm $(n=9)$. Cyan asterisks show cases that were expert-classified as $F S$ or non- $F S$, but as pyramidal neurons by the formal algorithm $(n=2)$. A3, B3, Classification of FS $(n=80)$ against non-FS $(n=51)$ neurons. Magenta, Cells that were expert-classified as FS, but attributed to non-FS class by the formal algorithm $(n=2)$. Cyan, Cells that were expert-classified as non-FS, but classified by the formal algorithm as FS ( $n=7)$.

from the threshold to the AHP amplitude measured from the threshold. From responses to depolarizing current steps, we measured coefficient of variation of interspike intervals (SD divided by the mean). We found, in correspondence with prior studies (Battaglia et al., 2013; Druckmann et al., 2013), that these four parameters have the strongest predictive power and therefore they were selected for classification of electrophysiological types of neurons of 19 parameters which we have measured and tested for formal classification (see Materials and Methods).

For the formal classification, we first trained an algorithm on typical representatives of FS $(n=53)$, non-FS $(n=22)$, and pyramidal $(n=39)$ neurons. Scatter plots illustrate clear separation of pyramidal neurons from inhibitory interneurons (Fig. 2A1), and FS from non-FS cells (Fig. 2B1). After training a linear discriminant model on this dataset, we run multivariate classification on all $(n=200)$ neurons ( $\mathrm{R}$ version 3.4.0, functions $l d a$ and predict; The R Foundation for Statistical Computing, 2017). In the first iteration, we discriminated between pyramidal neurons and interneurons. Scatter plots in Figure 2, A2 and B2, compare results of expert and formal classification. Results of expert and formal classification were the same in all but 11 cases: 2 expert-classified interneurons and 9 pyramidal neurons were attributed to a different class by the formal algorithm. All neurons classified as pyramidal by the expert and/or by the formal algorithm were excluded from the following analyses. After using these rigorous criteria of combined expert and formal classification, inhibitory neurons were classified as FS versus non-FS cells (Fig. 2A3,B3). Inhibitory neurons used for analyzing plasticity, were attributed to three groups as following: neurons for which results of expert and formal classification coincided, were attrib- uted to either FS (1) or non-FS (2) group; neurons for which expert and formal classification did not agree, were attributed to a mixed FS/non-FS group (3). Results of plasticity experiments were analyzed for (1) all inhibitory neurons including the latter Group 3, and (2) separately for the groups of FS and non-FS neurons.

\section{Plasticity of excitatory inputs to inhibitory neurons: pairing procedure induces changes at both paired and unpaired inputs}

We first ask whether a conventional pairing protocol (prebefore-post-STDP) induces plasticity at excitatory synapses to inhibitory neurons, and whether plastic changes are restricted to the stimulated synapses, or also occur in non-stimulated inputs. We used a weak STDP procedure consisting of 30 pairings of EPSPs followed by a burst of postsynaptic spikes with $10 \mathrm{~ms}$ interval. Figure $3 A$ shows an example of LTP of excitatory transmission induced in a Martinotti cell (Fig. 3A1) by the STDP procedure (Fig. 3A2). EPSP amplitude increased in this example from $1.53 \mathrm{mV}$ in control to $3.07 \mathrm{mV}$ after the pairing $(201 \%, p<$ $0.001)$. Potentiation persisted for the length of recording of this cell (60 min post-pairing; Fig. 3A3). Of 10 cells tested, 5 expressed significant LTP with average potentiation to $164 \pm 10 \%$ of control (mean \pm SEM, $n=5$ ). In two further cells LTD was observed, and in the remaining three neurons EPSP amplitude did not change after the pairing. When averaged over all tested neurons, responses in the paired inputs were potentiated to $129 \pm 13 \%$ of control $(n=10, t$ test, $p=0.0514)$. We observed LTP of the paired inputs to both FS and non-FS neurons. Notably, plastic changes were not restricted to the paired inputs. Rather, unpaired 
A1

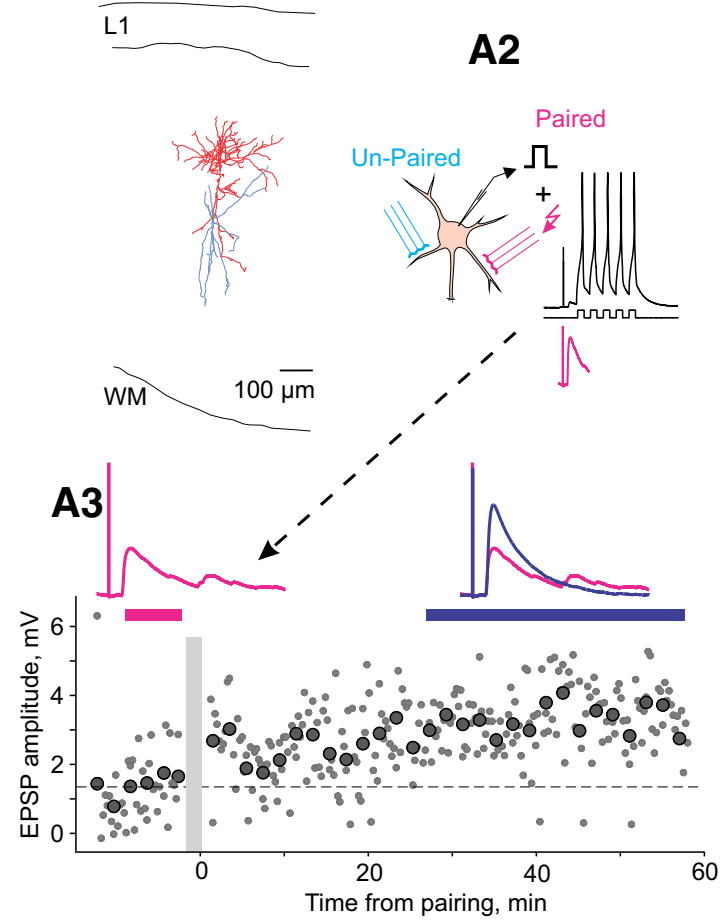

B
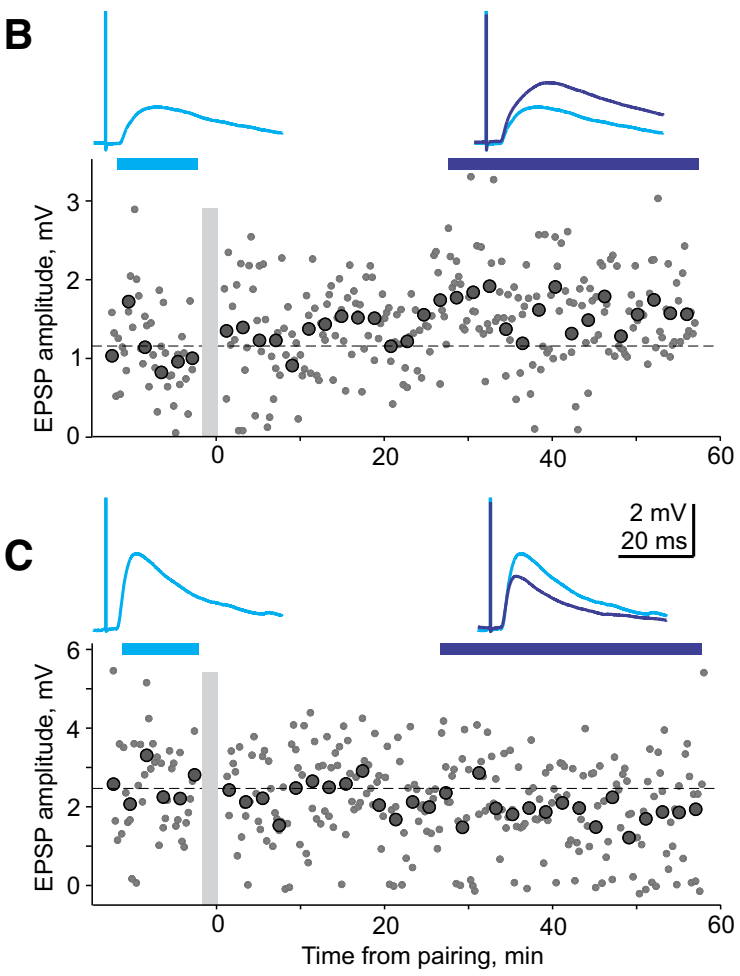

Figure 3. Pairing procedure (STDP protocol) induces plastic changes at both paired and unpaired excitatory synapses to inhibitory neurons in rat visual cortex. $A$, Plasticity of excitatory synaptic transmission in a Martinotti cell from rat visual cortex. A1, Reconstruction of the Martinotti cell, with dendrites in blue and axon in red. L1: layer 1; WM: white matter; A2, A scheme of pairing-induced plasticity experiment. Two independent test inputs to a neuron were stimulated in alternation, every $7.5 \mathrm{~s}$. A pre-before-post-STDP protocol, in which a burst of postsynaptic spikes (5 depolarizing pulses at $100 \mathrm{~Hz}$ ) was evoked $10 \mathrm{~ms}$ after presynaptic stimulation of one of the inputs (magenta). The second input (cyan) was not stimulated during plasticity induction. Pairing procedure was repeated 30 times. A3, LTP induced in the paired input. Response amplitudes (small symbols show individual responses; large symbols, averages over 2 min) plotted against time after the pairing (gray vertical bar). Horizontal dashed line shows average response amplitude during control period. Traces show averaged responses during the indicated periods before (magenta) and after (blue) the pairing. Data from the cell shown in $\boldsymbol{A 1}$. $\boldsymbol{B}, \boldsymbol{C}$, Examples of LTP $(\boldsymbol{B})$ and LTD $(\boldsymbol{C})$ induced in unpaired inputs to two inhibitory neurons. Non-stimulated inputs (cyan, as in $\boldsymbol{A 2}$ ) could express LTP or LTD after the pairing procedure (gray vertical bar) applied to other inputs to these neurons.

inputs could also express significant LTP (Fig. 3B) or LTD (Fig. $3 C)$. Despite significant LTP or LTD in individual inputs, responses to unpaired inputs averaged over all tested neurons did not show significant changes $(110 \pm 10 \%$ of control, $n=10, p=$ 0.29 ).

These results show that changes of responses averaged over all studied neurons are consistent with Hebbian-type input specific plasticity: potentiation at paired inputs after pre-before-postpairing, and no changes of the grand average of all unpaired inputs. However, plastic changes in individual excitatory inputs to inhibitory neurons substantially deviate from these general rules. First, in the paired inputs, a "potentiating" pre-before-post protocol could induce a depression, or do not lead to any changes at all. Second, in the unpaired inputs both potentiation and depression were observed, thus providing evidence for heterosynaptic changes occurring in parallel to input-specific plasticity.

One concern in experiments with extracellular stimulation is independence of tested inputs. If the sets of stimulated presynaptic fibers overlap, this may lead to "seemingly-heterosynaptic" changes that were actually mediated by changes in the synapses activated by both stimuli. Such a scenario predicts that "heterosynaptic" changes at unpaired inputs are of the same sign as changes in the paired inputs, but of a smaller magnitude. More generally, changes in occluding inputs to a cell should be positively correlated. These predictions did not hold for our dataset. In none of five cells in which paired inputs expressed LTP, the unpaired inputs were potentiated; in two cells expressing LTD in the paired inputs, one case of LTP and one case of LTD was observed in the unpaired inputs. Similar configuration of recording and stimulation electrodes was also used in experiments with intracellular tetanization (described in the next three sections), therefore we tested for correlation between synaptic changes in two test inputs (S1 and S2; see Materials and Methods, Experimental design: synaptic stimulation) to neurons in which both S1 and S2 fulfilled inclusion criteria (see Materials and Methods, Data processing and statistical analysis). No correlation between changes in the two inputs was found in these neurons $(r=0.072, n=90, p>0.5)$, indicating that there was no significant overlap between two tested inputs to the same neuron in our experiments. Thus, changes at unpaired inputs were indeed heterosynaptic.

\section{Heterosynaptic plasticity of unitary excitatory inputs to inhibitory neurons}

To verify that heterosynaptic plasticity observed in the above experiments indeed could be induced without presynaptic activation we used recordings from monosynaptically connected neuron pairs. This allowed us to monitor activity of both presynaptic (excitatory) and postsynaptic (inhibitory) neurons, and control for a possibility of occasional presynaptic spikes which may potentially lead to a "spurious" pairing (Abraham et al., 2007). In these experiments we used protocol of intracellular tetanization, established in our prior studies of plasticity in pyramidal neurons (Volgushev et al., 2000; Chen et al., 2013; Chistiakova et al., 2015; Bannon et al., 2017). Our prior work showed that, in pyramidal neurons, intracellular tetanization induces 

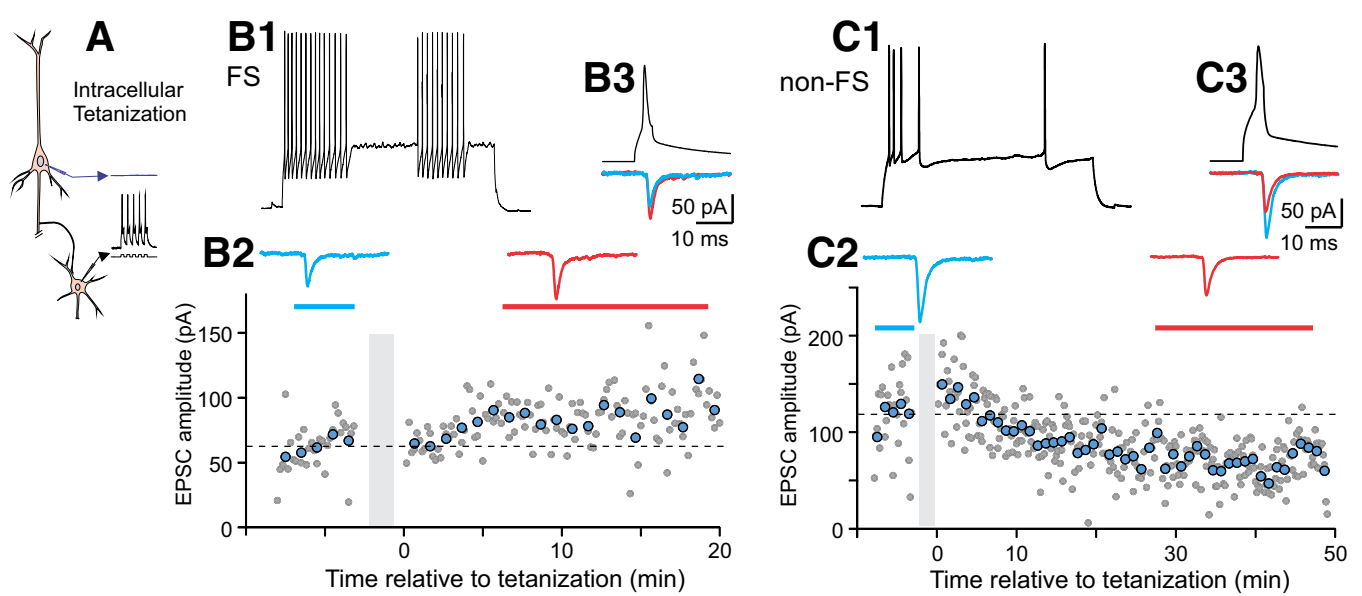

Figure 4. Intracellular tetanization induces long-term plasticity of unitary EPSCS in inhibitory neurons. A, A scheme of intracellular tetanization experiment in paired recordings. Intracellular tetanization consisted of 30 bursts of 5 spikes induced in the postsynaptic cell by short depolarizing pulses $(5 \mathrm{~ms}, 100 \mathrm{~Hz})$. No spikes occurred during intracellular tetanization in the presynaptic neuron. B1-B3, Potentiation of unitary excitatory responses in a FS neuron from layer 3 after intracellular tetanization. B1, Firing pattern of the postsynaptic FS neuron. B2, Time course of unitary EPSC amplitude changes before and after intracellular tetanization, gray circles are individual amplitudes, larger blue circles are averages over 1 min. Horizontal dotted line shows mean response amplitude before tetanization. Averaged EPSCs are shown from the indicated periods before and after tetanization. $\boldsymbol{B}$ 3, Superimposed averaged EPSCs from $\boldsymbol{B 2}$, together with an average of presynaptic spikes. C1-C3, Depression of unitary EPSCs in a non-FS neuron from layer 3 after intracellular tetanization. C1, Firing pattern of the postsynaptic non-FS neuron. C2, Time course of unitary EPSC amplitude changes and averaged responses before and after tetanization. Same conventions apply as in B2. C3, Superimposed EPSCs from C2, and an average of presynaptic spikes.

strong calcium influx, with the magnitude proportional to the number of APs (Balaban et al., 2004). This rise in intracellular calcium concentration is necessary for the induction of heterosynaptic plasticity, because plasticity is blocked if the calcium chelator EGTA $(10 \mathrm{~mm}$ ) is added to the pipette solution (Lee et al., 2012; Volgushev et al., our unpublished data). We propose that in inhibitory neurons intracellular tetanization leads to a similar influx of calcium, which then triggers heterosynaptic plasticity.

Intracellular tetanization mimics the conditions which unpaired inputs experience during the pairing procedure. It consisted of 30 bursts of 5 APs induced by bursts of short depolarizing pulses in the postsynaptic neuron, without presynaptic stimulation. Figure $4 A$ shows an example of one burst of spikes evoked in the postsynaptic inhibitory neuron, and absence of spikes in the presynaptic pyramidal neuron. In this and in all other experiments with paired recordings, no spikes in the presynaptic neuron occurred during intracellular tetanization.

Figure $4 B$ shows example of LTP induced by intracellular tetanization in a connection between pyramidal cell and a FS interneuron from layer 3. EPSCs recorded in the FS interneuron increased from $62 \pm 2.9 \mathrm{pA}$ in control to $86 \pm 3.1 \mathrm{pA}$ after intracellular tetanization (potentiation to $139 \%$ of control; $p<$ $0.001)$. In another experiment, intracellular tetanization induced LTD in a connection from pyramidal neuron to a non-FS neuron from layer 3 (Fig. 4C). EPSC amplitudes in the non-FS neuron were reduced from $118 \pm 6.8 \mathrm{pA}$ in control to $74 \pm 2.2 \mathrm{pA}$ (depression to $63 \%, p<0.001$ ). Of the $n=16$ unitary pyramidto-interneuron connections tested in paired recordings, intracellular tetanization led to plastic changes in 10 cases ( 7 cases of potentiation and 3 cases of depression).

These experiments demonstrate that intracellular tetanization could induce lasting changes at unitary excitatory connections to inhibitory neurons. The observed plasticity was clearly heterosynaptic, as it occurred at synapses that were not activated during the induction.

Although paired recordings provide a clear case for heterosynaptic plasticity, technical difficulty of plasticity experiments with connected pairs of neurons makes such experiments impractical for collecting large data samples. Therefore for the main experimental series with intracellular tetanization, we opted to use EPSPs evoked in inhibitory neurons by extracellular stimulation.

\section{Heterosynaptic plasticity of pharmacologically isolated excitatory inputs to inhibitory neurons}

One drawback of extracellular stimulation is that, in line with activating synaptic inputs to the recorded neuron, it also activates unknown number of other neurons in the slice. Because the subject of this study is plasticity of excitatory synapses on inhibitory neurons, it was important to exclude possible contribution of changes of inhibition. Although inhibition did not contribute to the studied synaptic responses directly, as verified by depolarization/reversal test, extracellular stimulation might activate inhibitory neurons that do not make synapses on the recorded postsynaptic cell, but still release GABA. To confirm that observed plastic changes of excitatory responses are not contaminated by changes of inhibition, we performed a series of experiments with a $\mathrm{GABA}_{\mathrm{A}}$-receptor blocker picrotoxin $(50 \mu \mathrm{M})$ added to the extracellular solution.

Figure 5 shows a scheme of intracellular tetanization experiments using extracellular stimulation (Fig. 5A) and two examples of LTP (Fig. $5 B, C$ ). In the first example, the amplitude of EPSP in a FS cell increased from $0.77 \mathrm{mV}$ in control to $1.17 \mathrm{mV}$ after intracellular tetanization, thus expressing an LTP of 152\% (Fig. $5 B$ ). Another input to that neuron tested in the same experiment did not express significant changes after intracellular tetanization (amplitude $91 \%$ of control, data not shown). In the second example, an input to another FS cell expressed LTD, with the amplitude of EPSP decreasing to $55 \%$ of control after the tetanization, from 3.22 to $1.76 \mathrm{mV}$ (Fig. $5 C$ ). Of the 37 pharmacologically isolated EPSPs tested in this series, intracellular tetanization led to LTP in 9 (24\%), LTD in $14(38 \%)$ inputs and did not induce significant changes of EPSP amplitude in 14 (38\%) inputs. These results show that intracellular tetanization could induce bidirectional heterosynaptic plasticity in the presence of $\mathrm{GABA}_{\mathrm{A}}$ receptor blocker PTX.

Our prior work demonstrated that one of the determinants of the direction of heterosynaptic changes is initial PPR, which is a 

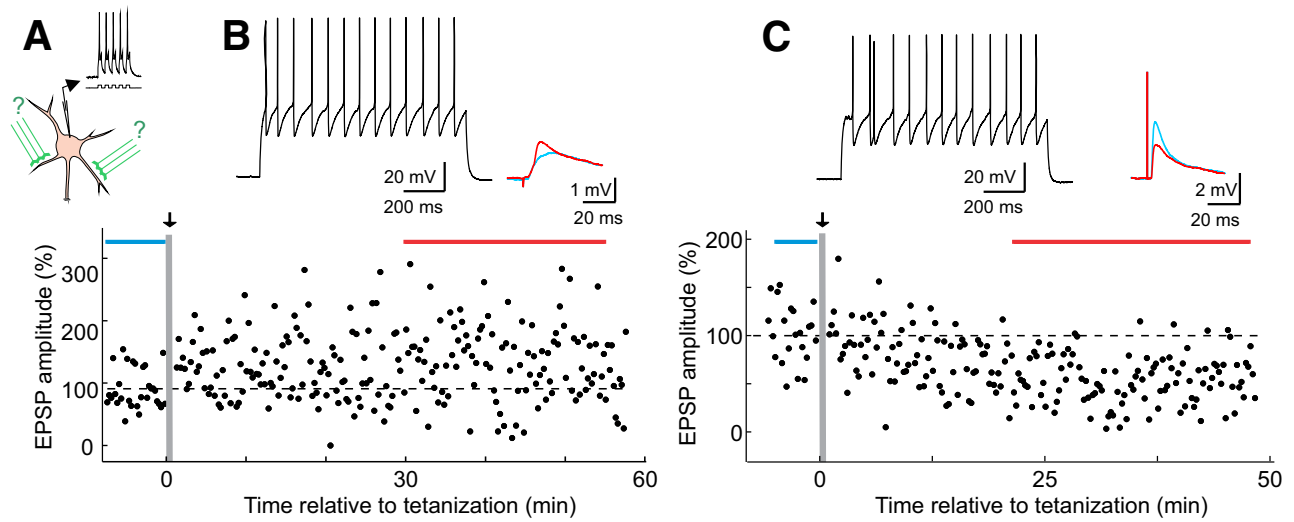

Figure 5. Intracellular tetanization induces long-term plasticity of pharmacologically-isolated EPSPs in inhibitory neurons. $A$, A scheme of intracellular tetanization experiment. In these experiments, $50 \mu \mathrm{M}$ PTX was added to the extracellular medium to isolate excitatory inputs to inhibitory neurons. $\boldsymbol{B}, \boldsymbol{C}$, Example LTP $(\boldsymbol{B})$ and LTD (C) induced in FS neurons by intracellular tetanization. For each example neuron, figure panels show firing pattern in response to $1 \mathrm{~s}$ depolarization current, time course of response amplitude changes, and EPSPs averaged over indicated periods before and after the tetanization. Time of intracellular tetanization is indicated by vertical gray bar in the time course.

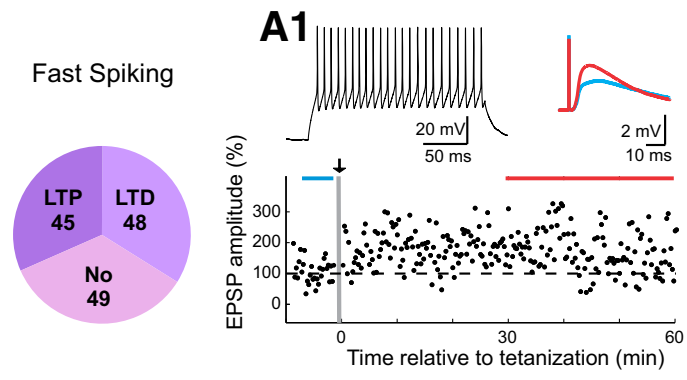

B1

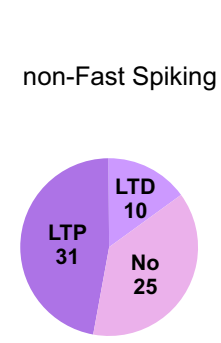

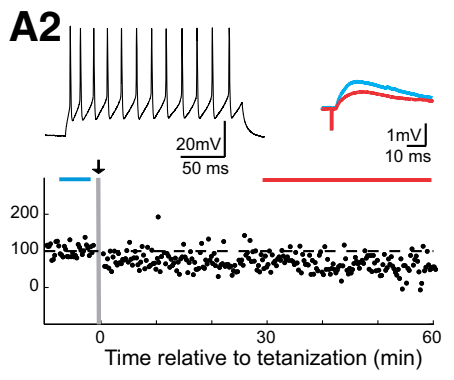

B2

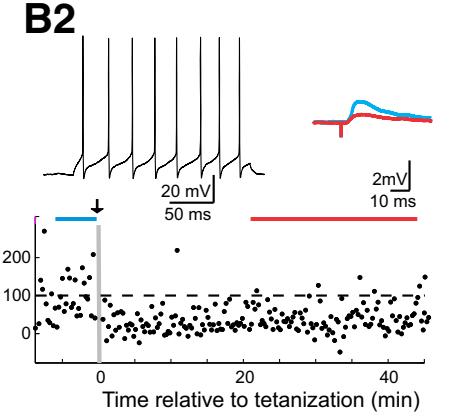

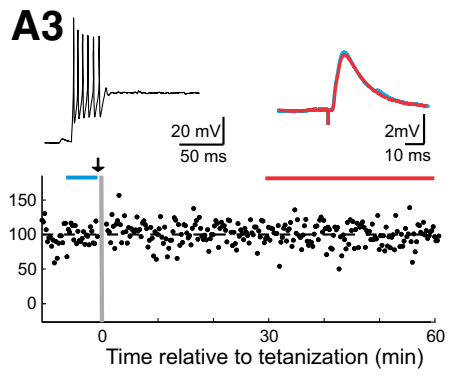

B3

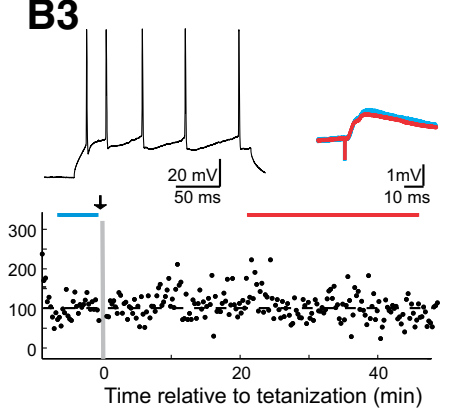

Figure 6. LTP induced in FS and non-FS inhibitory neurons by intracellular tetanization. $\boldsymbol{A}, \boldsymbol{B}$, Examples of synaptic responses in $F S(\boldsymbol{A} \mathbf{1}-\boldsymbol{A} \mathbf{3})$ and non-FS (B1-B3), which expressed LTP $(\boldsymbol{A} 1, \boldsymbol{B} \mathbf{1})$, LTD $(A 2, B 2)$, or did not change after intracellular tetanization $(\boldsymbol{A} \mathbf{3}, \boldsymbol{B} 3)$. Each panel shows pattern of response to a long depolarizing pulse, time course of EPSP amplitude changes, and averaged EPSP before and after tetanization, as indicated by color code. Pie charts show, for FS and non-FS cells, frequency of occurrence of LTP, LTD, and no changes after intracellular tetanization, and number of inputs contributing to each group.

correlate of presynaptic release mechanisms. However, electrical stimulation in the presence of blockers of $\mathrm{GABA}_{\mathrm{A}}$-mediated inhibition tends to evoke polysynaptic responses, which become especially pronounced with paired-pulse stimulation used in our study. Indeed, in 9 of 37 inputs included in the above analysis, responses to the second pulse in the paired-pulse stimulation paradigm were strongly contaminated by polysynaptic responses evoked by the first pulse, thus making the calculation of the PPR unfeasible. Therefore, we opted to do experiments in control solution, without blocking the $\mathrm{GABA}_{\mathrm{A}}$-receptors, and testing for excitatory nature of recorded responses using depolarization/reversal test (see Materials and Methods).

\section{Heterosynaptic plasticity of excitatory inputs to FS and non-FS neurons}

To induce heterosynaptic plasticity we used the protocol of intracellular tetanization, established in our prior work on pyramidal neurons (Volgushev et al., 2000; Chen et al., 2013; Chistiakova et al., 2015; Bannon et al., 2017) and verified for excitatory inputs to inhibitory interneurons in paired-recording experiments (Fig. 4) and in experiments with presence of $\mathrm{GABA}_{\mathrm{A}}$ blocker PTX (Fig. 5).

Figure 6 shows examples of LTP induced by intracellular tetanization in FS and non-FS cells. In cells of both types, intracellular tetanization could induce LTP (Fig. 6A1,B1) or LTD (Fig. $6 A 2, B 2)$, or did not change synaptic transmission at excitatory synapses to these cells. The frequency of occurrence of LTP and LTD was, however, significantly different in FS and non-FS cells (Fig. 6, pie charts). In the $n=142$ inputs to $n=93$ FS neurons tested, intracellular tetanization could lead to about equal number of cases of LTP, LTD, or no changes. $N=45$ inputs (32\%) expressed LTP, $n=48$ inputs (34\%) expressed LTD, and in $n=$ 49 inputs (34\%) EPSP amplitudes did not change. In contrast, in $n=66$ tested inputs to $n=39$ non-FS neurons intracellular 

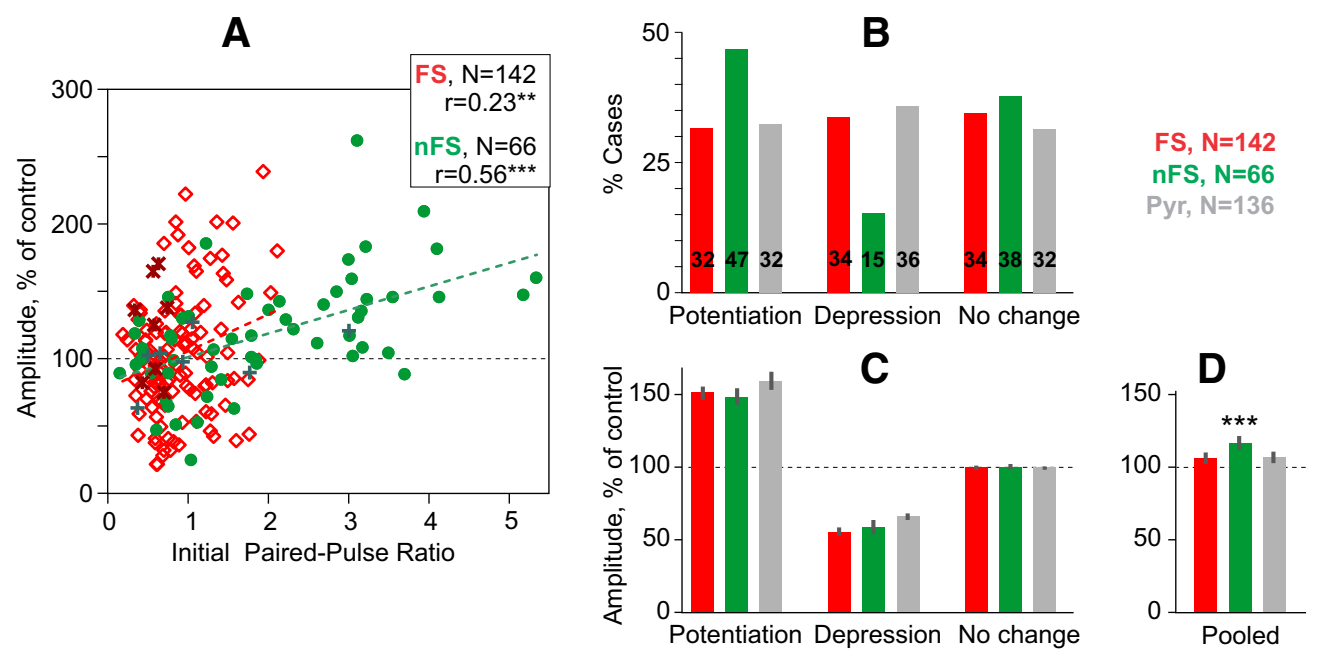

Figure 7. Distinct PPR and heterosynaptic plasticity in excitatory synapses to FS and non-FS interneurons. $A$, Changes of EPSP amplitude after intracellular tetanization plotted against initial PPR for inputs to FS (red diamond symbols; $n=142$ ) and non-FS (green circles; $n=66$ ) neurons. In both groups of cells, correlations between initial PPR and EPSP amplitude changes were significant $\left({ }^{*} p<0.05,{ }^{* * *} p<0.001\right)$. Data for unitary responses from paired recordings are shown as dark red asterisks ( $F S ; n=8$ ) and dark green crosses (non-FS; $\left.n=8\right)$. Note that for excitatory inputs to FS cells $P P R<2$ was typical, whereas in non-FS neurons PPR $>2$ were frequently observed. $B$, Frequency of occurrence of LTP, LTD, and no changes in FS and non-FS cells. Numbers within bars show percentage of cases. Note the more frequent occurrence of LTP, and less frequent LTD in non-FS cells compared with FS cells or pyramids (Pyr). $C$, Magnitude of potentiation and depression in the inputs that expressed significant LTP or LTD. Note that the magnitude of LTP and LTD was similar in FS, non-FS, and in pyramidal neurons. D, Net changes of EPSP amplitude after intracellular tetanization in all FS $(n=142)$, non-FS $(n=66)$ and pyramidal $(n=136)$ neurons. Note the significant potentiation in non-FS cells $(* * *<0.001)$. In B-D Data for pyramidal neurons (from Volgushev et al., 2016) are shown for comparison.

tetanization led to LTP more often [31/66 inputs (47\%)] than to LTD [10/66 inputs (15\%)]. In 25 of 66 inputs (38\%) to non-FS neurons amplitude of EPSPs did not change after intracellular tetanization. The difference between FS and non-FS neurons in the frequency of occurrence of LTP, LTD, or no changes was significant $\left(\chi^{2}\right.$ score $\left.=8.64, p=0.013\right)$.

Factors determining the direction of heterosynaptic plasticity of excitatory inputs to inhibitory neurons

In both FS and non-FS neurons the direction and the magnitude of synaptic changes were significantly correlated with initial PPR (Fig. 7A; FS: $r=0.23, n=142, p=0.0059$; non-FS: $r=0.56, n=$ $66, p<0.0001$; all inhibitory neurons, including FS, non-FS, and mixed FS/non-FS group pooled together: $r=0.34, n=233, p<$ $0.0001)$. PPR, calculated as the ratio of averaged amplitudes of responses to the second and the first pulses applied with a $50 \mathrm{~ms}$ interval, is inversely related to the release probability, and is broadly used to assess the state of presynaptic release mechanisms (Voronin, 1993; Murthy et al., 1997; Oleskevich et al., 2000). Correlation of LTP changes with initial PPR indicates that after intracellular tetanization, inputs with initially lower release probability (high PPR) tended to underdo LTP, whereas inputs with initially higher release probability (low PPR) tended to undergo LTD or did not change.

To further evaluate possible factors that may determine heterosynaptic plasticity we analyzed a linear model, in which the change of EPSP amplitude after intracellular tetanization was considered a response, and cell type (FS or non-FS), layer, animal age on a day of slice preparation, and parameters of EPSP in control (amplitude, slope, latency, and PPR) were considered predictors. In the model which included all seven of the above predictors $\left(F_{\mathrm{DF}(7,159)}=6.218, p<0.001\right)$ the strongest predictor was the initial PPR $(t=4.989, p<0.001)$, followed by the EPSP slope $(t=2.715, p=0.0074)$. Predictive power of the remaining factors (cell type and layer, animal age, EPSP amplitude, and latency) was not significant ( $p>0.1$; function $l m$, R version 3.4.0;
The R Foundation for Statistical Computing, 2017). For subsets of each size the optimal combination of predictors that minimized the residual error (function regsubsets in the library leaps) always included the PPR. EPSP slope was the second-most frequent predictor. Interestingly, there was no significant correlation between EPSP amplitude change and initial slope for the whole sample $(r=-0.026, n=233, p>0.1)$ as well as for FS neurons $(r=0.124, n=142, p>0.1)$; however, within the group of non-FS neurons there was a significant negative correlation $(r=-0.31, n=66, p=0.0102)$.

Although cortical layer was not a significant predictor of EPSP amplitude change after intracellular tetanization, there were significant correlations between EPSP amplitude change and layer for the groups of non-FS neurons $(r=0.31, n=66, p=0.0116)$ and FS neurons $(r=0.173, n=142, p=0.0395)$ when considered separately (Fig. $8 A$ ). For all inhibitory neurons pooled together, however, there was no significant correlation $(r=0.104$, $n=233, p=0.113$ ).

There was no significant correlation between animal age and heterosynaptic plasticity. Scatter plot of EPSP amplitude change against age did not reveal age dependence (Fig. $8 B$ ), and correlation coefficients for FS neurons $(r=0.011, n=142)$, non-FS neurons $(r=-0.176, n=66)$, and all inhibitory neurons pooled $(r=-0.009, n=233)$, were nonsignificant $(p>0.1$ for all 3$)$. We note here that studying age dependence of heterosynaptic plasticity was of scope of present study, and the results were obtained chiefly from animals between P19 and P30 [206/233 inputs $(88 \%)$ ].

The above analyses revealed that, among the analyzed parameters, the initial PPR was the strongest predictor of the direction and magnitude of EPSP amplitude changes after intracellular tetanization. We refer to the correlation between initial PPR and direction of heterosynaptic changes as "weight-dependence" of plasticity (Volgushev et al., 1997; Chistiakova et al., 2015; Bannon et al., 2017). This terminology follows experimental studies that established weight-dependence of synaptic plasticity in cultured 
A

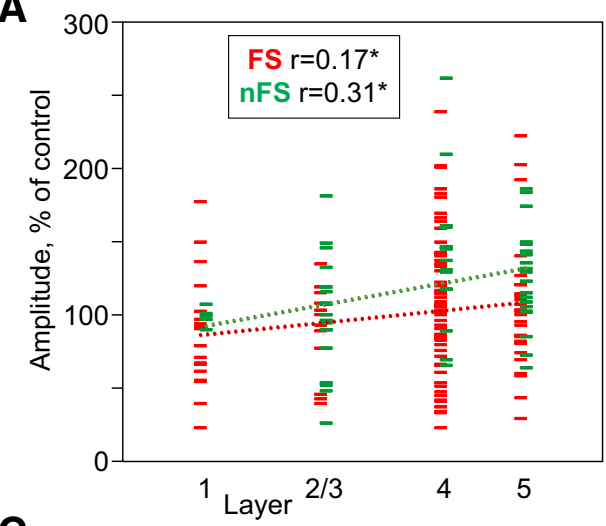

C

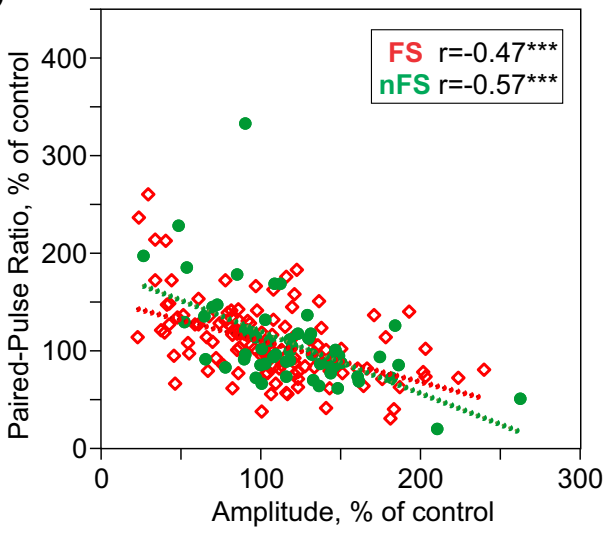

B

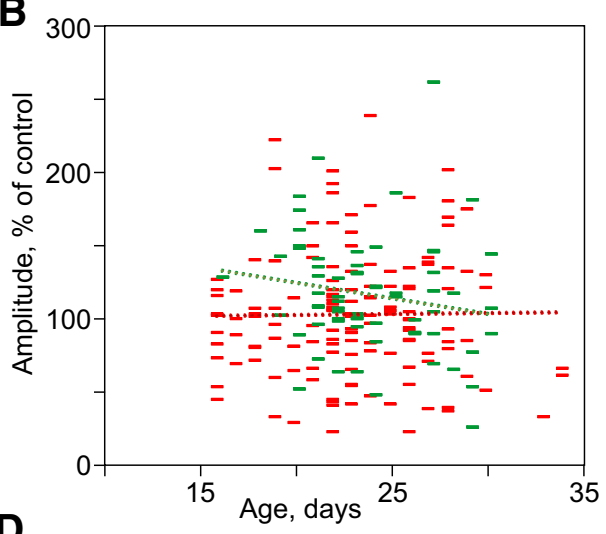

D

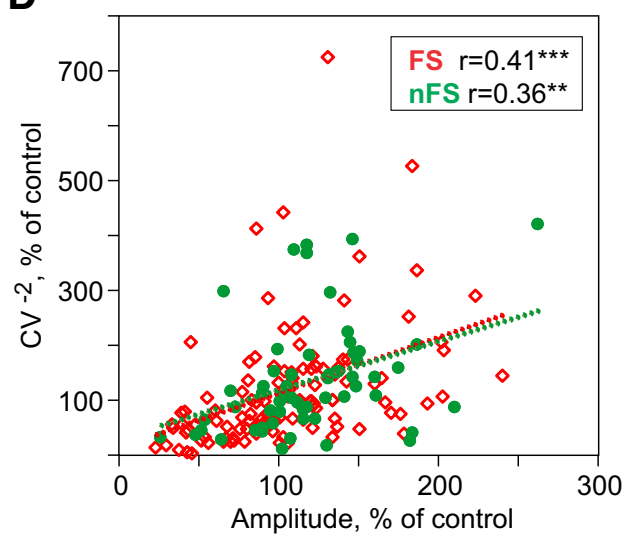

Figure 8. Properties of heterosynaptic plasticity of excitatory transmission to inhibitory neurons induced by intracellular tetanization. Each point represents data for one tested synaptic input to FS (red diamonds or dashes; $n=142$ ) or non-FS (green circles or dashes; $n=66$ ) cells. $\boldsymbol{A}, \boldsymbol{B}$, Heterosynaptic plasticity involves presynaptic mechanisms. In both, FS and non-FS neurons, changes of EPSP amplitude after intracellular tetanization were significantly correlated with changes of the PPR $(\boldsymbol{A})$ and changes of the $\mathrm{CV}^{-2}(\boldsymbol{B})$. $\boldsymbol{C}$, Changes of EPSP amplitude after intracellular tetanization in inhibitory neurons from different layers. For each layer, data points are slightly shifted to the left for FS neurons (red), and to the right for non-FS neurons (green). Significant correlation was observed only for non-FS neurons. D, Changes of EPSP amplitude after intracellular tetanization in inhibitory neurons recorded in slices from animals of different age. For each day, data points are slightly shifted to the left for FS neurons (red), and to the right for non-FS neurons (green). Neither in FS nor in non-FS neurons were the EPSP amplitude changes correlated with age. $\boldsymbol{A}-\boldsymbol{D}$, Dotted lines show linear regression trend lines; correlation coefficients are shown for significant correlations only, with significance denoted as ${ }^{*} p<0.05,{ }^{* *} p<0.01,{ }^{* * *} p<0.001$.

hippocampal neurons (Bi and Poo, 1998; Debanne et al., 1999). Synaptic weight in these studies was measured as amplitude of averaged EPSPs, which is a product of release probability, quantal size and the number of release sites. In our study we measured a correlate of synaptic weight, PPR, which is inversely proportional to the release probability and thus to synaptic weight. Note that synaptic weight defined as above combines "reliability" of a synapse (determined by presynaptic release probability) and "synaptic efficacy” (postsynaptic effect of released transmitter), which are often considered separately.

\section{Distinct features of synaptic transmission and heterosynaptic plasticity in FS and non-FS neurons}

Excitatory transmission to FS and non-FS neurons differed, however, in several important aspects.

First, in control, before intracellular tetanization, inputs to non-FS neurons expressed significantly higher PPRs than inputs to FS neurons $(1.90 \pm 0.16$, range $0.15 \ldots 5.3, n=66$ non-FS vs $0.9 \pm 0.03$; range $0.17 \ldots 2.1, n=142 \mathrm{FS} ; p<0.0001)$. This conclusion is supported by analysis of a linear model, in which the initial PPR was considered a response, and measured electrophysiological parameters characterizing cell type (cell type, FS, or non-FS; the amplitude, AHP, and width of APs) and postsynaptic response (slope and latency) were considered predictors (function $l m$, R version 3.4.0; The R Foundation for Statistical Computing, 2017). Cell type was the strongest predictor of the PPR $(t=4.72, p<0.001)$. For subsets of each size, the optimal combination of predictors that minimized the residual error (function regsubsets in the library leaps) always included cell type. Further, initial PPR strongly correlated with cell type $(r=$ $-0.342, n=201, p<0.001)$. Next strongest predictor of initial PPR was the slope of EPSP $(t=-3.87, p<0.001)$. Possible reason for this relation could be that EPSP slope was correlated with cell type ( $r=-0.184, n=208, p=0.0078)$, and was different between FS and non-FS neurons $(1.04 \pm 0.07 \mathrm{mV} / \mathrm{ms}$ in FS, vs $0.74 \pm 0.07$ in non-FS, $p=0.013$ ).

Second, inputs to non-FS neurons expressed LTP more frequently, and LTD less frequently, than inputs to FS neurons (Fig. $7 B$ ). LTP was observed in $47 \%$ of inputs to non-FS cells ( 31 of 66 ), but in $32 \%$ of inputs to FS neurons ( 45 of 142). LTD occurred in $15 \%$ of inputs to non-FS cells (10/66) but in $34 \%$ of inputs to FS neurons (48/142). For comparison, in pyramidal neurons (data from our prior study, Volgushev et al., 2016), frequency of occurrence of LTP (32\% of cases) and LTD (36\% of cases), was similar to that in FS neurons but significantly different from the frequency of LTP and LTD in non-FS neurons (Fig. 7B).

Third, the net effect of intracellular tetanization on excitatory synaptic transmission to non-FS neurons was potentiation to $116 \pm 4.9 \%$ of control $(n=66, p<0.001)$. This net potentiation stands in a marked contrast to balanced synaptic changes induced by intracellular tetanization in FS neurons (106 $\pm 3.7 \%$ of con- 
trol, $n=142, p>0.1)$ or in pyramidal neurons $(106 \pm 4 \%$ of control, $n=136, p>0.1$; data from Volgushev et al., 2016; Fig. $7 D)$. Note that this difference in the net outcome of heterosynaptic plasticity induced by intracellular tetanization was because of the more frequent occurrence of LTP in non-FS interneurons, and not to a larger magnitude of LTP in individual connections. The magnitude of potentiation at individual inputs was not different in FS ( $151 \pm 4.6 \%$ of control, $n=45$ cases of LTP) and non-FS neurons ( $149 \pm 5.6 \%, n=31$ cases of LTP; Fig. $7 C)$. Also, magnitude of significant LTD was similar: inputs to FS cells were depressed to $56 \pm 3.1 \%$ of control ( $n=48$ cases of LTD) and inputs to non-FS cells were depressed to $59 \pm 4.8 \%$ of control $(n=10$ cases of LTD).

One further difference between FS and non-FS cells was the correlation of EPSP amplitude changes after intracellular tetanization and EPSP slope, mentioned in the section Factors determining the direction of heterosynaptic plasticity above. In non-FS neurons, EPSP amplitude changes were negatively correlated with the initial EPSP slope $(r=-0.31, n=66, p=0.0102)$. In FS neurons there was no such correlation $(r=0.124, n=142$, $p>0.1)$.

\section{Heterosynaptic plasticity in FS and non-FS neurons involves presynaptic mechanisms}

EPSP amplitude changes induced by intracellular tetanization were associated with changes of two indices of presynaptic release: PPR and inversed coefficient of variation $\left(\mathrm{CV}^{-2}\right)$. In both FS and non-FS neurons, changes of the PPR were negatively correlated with the changes of response amplitude in FS neurons $(r=-0.47, p<0.001)$, non-FS neurons $(r=-0.57, p<0.001$; Fig. $8 C)$, and for all inhibitory neurons pooled together $(r=$ $-0.50, p<0.001)$. Changes of the inverse $\mathrm{CV}^{-2}$ were positively correlated with response amplitude changes (Fig. $8 D ; r=0.41$, $p<0.001$ for FS; $r=0.36, p=0.003$ for non-FS; and $r=0.43, p<$ 0.001 for pooled interneurons). Changes of PPR and changes of $\mathrm{CV}^{-2}$ were significantly correlated for FS $(r=-27, p=0.0011)$, non-FS $(r=-0.35, p=0.0044)$, and for the whole sample of interneurons $(r=-0.31, p<0.001)$ demonstrating that two presynaptic indices changed coherently. The conclusion on involvement of presynaptic mechanisms in heterosynaptic plastic changes is supported by analysis of the linear model that considers EPSP amplitude change a response (dependent variable) and cell type, layer, animal age, initial EPSP parameters (amplitude, slope, latency and PPR), and changes of PPR and $\mathrm{CV}^{-2}$ as predictors. Changes of PPR and changes of $\mathrm{CV}^{-2}$ were the strongest "predictors" of EPSP amplitude changes $(t=-4.029, p<0.001$; and $t=3.683, p<0.001$, respectively, function $l m$ in R). Next strongest predictor of EPSP amplitude change was initial PPR $(t=3.635, p<0.001)$, consistent with the weight-dependence of heterosynaptic plasticity described above. These three factors were present in all (PPR-change), all but one $\left(\mathrm{CV}^{-2}\right.$ change) and all but two (initial PPR) subsets minimizing the residual error (function regsubsets in the library leaps). Note that neither laminar location nor age were significant predictors of EPSP amplitude change in this model $(t=-0.11$ and $t=-0.01 ; p>0.9$ for both).

The above analyses indicate that potentiation was associated with an increase, and depression with a decrease of release probability, and thus heterosynaptic plasticity was partially expressed presynaptically. Furthermore, correlation between PPR change and initial PPR $(r=-0.344, p<0.001$ for FS; $r=-0.479, p<$ 0.001 for non-FS; $r=-0.285, p<0.001$ for pooled interneurons) suggests that involvement of presynaptic mechanisms in expression of heterosynaptic LTP or LTD correlated with the initial state of release mechanisms.

\section{Discussion}

We describe a novel form of plasticity of excitatory synapses on inhibitory neurons; weight-dependent heterosynaptic plasticity. Recordings from connected pyramid-to-interneuron pairs confirm that purely-postsynaptic protocols without spikes in the presynaptic cell can induce plasticity, which is thus heterosynaptic. Heterosynaptic changes accompany homosynaptic plasticity induced by conventional STDP-protocols. In both FS and non-FS neurons heterosynaptic plasticity was weight-dependent.

\section{Homosynaptic plasticity in inhibitory neurons}

Prior research demonstrated that excitatory synapses on inhibitory neurons are plastic, and revealed highly heterogeneous rules and conditions for induction of plasticity in diverse types of interneurons.

In the hippocampal CA1, plasticity of excitatory synapses on FS interneurons from stratum pyramidale lacked the inputspecificity, and both homo and heterosynaptic sites could express LTP, LTD, or no changes (Cowan et al., 1998). In contrast, excitatory synapses to interneurons in strata radiatum and oriens expressed strictly input-specific plasticity, either Hebbian-type in stratum radiatum, or "anti-Hebbian" in stratum oriens (Lamsa et al., 2005, 2007).

In mouse visual or somatosensory cortex, excitatory inputs to FS neurons expressed mGluR5-dependent LTP in control conditions (Sarihi et al., 2008) or only in the presence of $\alpha 1$ and $\beta$ adrenergic agonists (Huang et al., 2013). No LTP was found in non-FS interneurons (Sarihi et al., 2008). However, in SSTexpressing neurons, a NMDA-independent LTP was found in control (Chen et al., 2009), and regular STDP when both adrenergic and NMDA-receptors were activated (Huang et al., 2013). In somatosensory cortex of rat pups, regular STDP was found in LTS (non-FS) neurons, whereas only LTD could be induced in FS cells (Lu et al., 2007). Strong potentiation of L4 star pyramid connections to FS neurons was found after visual deprivation (Maffei et al., 2006), though detail of activity patterns inducing this plasticity remain undefined.

Our results add novel observations to this mosaic, showing that excitatory inputs to both FS and non-FS neurons can express LTP after pairing procedure. The vast diversity of experimental conditions: species (rats, mice), age (P13-P56), cortical regions (hippocampus, visual, somatosensory), and induction protocols (afferent tetanization, pairing or deprivation during development) prevents across-the-board comparisons between existing studies. Although further research is needed to clarify all the factors underlying the observed diversity of plasticity rules, one emerging factor is the identity of presynaptic and postsynaptic neurons. Plasticity rules can be cell-type-specific, and even connection-specific (Larsen and Sjöström, 2015). Dependence of heterosynaptic plasticity on initial PPR revealed in the present study supports that latter proposition, indicating that initial state of release mechanisms is a crucial factor contributing to the observed diversity of plasticity at excitatory synapses to inhibitory neurons.

\section{Heterosynaptic plasticity in inhibitory neurons}

We found that heterosynaptic plasticity can be induced at excitatory synapses to inhibitory neurons and is weight-dependent. These results extend our prior work on heterosynaptic plasticity in pyramidal neurons (Volgushev et al., 2000; Lee et al., 2012; 
Chen et al., 2013; Volgushev et al., 2016; for review, see Chistiakova et al., 2014, 2015) in several important ways.

Paired recordings from pyramid-to-interneuron connections provide a clear demonstration of heterosynaptic plasticity. Longterm changes could be induced by purely-postsynaptic intracellular tetanization with documented absence of spikes in the presynaptic cell during the induction. Interestingly, a developmental study of plasticity in layer 4 of visual cortex found that in reciprocally-connected FS interneurons and star pyramids $(n=$ 7), high-frequency firing of FS neuron induced LTP in pyramidto-FS synapses in slices from P22-P23 rats (Lefort et al., 2013). Note that induction protocol used by Lefort et al., 2013 (10 APs at $20 \mathrm{~Hz} ; 10$ times at $0.1 \mathrm{~Hz}$ ) was similar to intracellular tetanization which we used. Heterosynaptic changes at unpaired inputs during STDP-pairing confirm our conclusion from studying L2/3 pyramids that heterosynaptic changes are induced by conventional plasticity-induction protocols (Volgushev et al., 2016).

Moreover, despite a broad variety of interneuron types (Battaglia et al., 2013; Druckmann et al., 2013) and type-specific rules for homosynaptic plasticity (Lu et al., 2007; Sarihi et al., 2008; Chen et al., 2009; Huang et al., 2013), we found weightdependence of heterosynaptic plasticity, both within FS and non-FS types, and in all interneurons pooled together. Together with our prior work on pyramidal neurons and computer simulations (Chen et al., 2013) the present results demonstrate that weight-dependent heterosynaptic plasticity is a widespread phenomenon, which could play a role of universal homeostatic "brakes" preventing runaway dynamics at excitatory synapses (Chistiakova et al., 2015). Such constraints allow learning networks to benefit from a broad variety of plasticity rules, STDP windows, and activity patterns, while at the same time robustly maintaining stable regime of operation.

The weight-dependence of heterosynaptic changes has one further important implication. It demonstrates that excitatory synapses at inhibitory neurons exhibit dispositions for potentiation or depression, much like synapses at pyramidal neurons do (Volgushev et al., 1997). Because synaptic weight and release probability are products of prior plastic changes, the dispositions reflect the dependence of plasticity on the history of changes; metaplasticity (Abraham and Bear, 1996).

Interestingly, although heterosynaptic plasticity was weightdependent in all interneurons, it had different net effect in FS and non-FS cells. In FS neurons, heterosynaptic changes were balanced: the gross average of amplitude changes in $n=142$ inputs was not different from pre-induction control. In contrast, non-FS neurons ( $n=66$ inputs) expressed net potentiation. This difference could be because of higher initial PPR in non-FS neurons, observed here and consistently reported in prior studies (Silberberg et al., 2005; Lu et al., 2007). Difference in initial PPR and in net effects of heterosynaptic changes after episodes of strong activity may be related to diverse network roles of FS and non-FS neurons.

Most FS neurons are PV-expressing basket and chandelier cells targeting pyramidal neurons and controlling horizontal spread of activity (Bacci et al., 2005; Silberberg et al., 2005; Hu et al., 2014; Jiang et al., 2015; Cardin, 2018). Low PPR at excitatory inputs to FS neurons accentuates their response to the first spike in a train (Tsodyks and Markram, 1997). As a result, precisely synchronized inputs activate FS neurons effectively and rapidly. Indeed, FS neurons are involved in generation of high-frequency oscillations and maintenance of large-scale excitation/inhibition balance in neuronal networks (Cardin et al., 2009; Sohal et al., 2009; Allen and Monyer, 2015). Balanced heterosynaptic changes in FS neurons would help to keep overall excitation/inhibition balance and balanced regime of operation of cortical networks, while allowing for local redistribution of activity and synchronization.

Non-FS cells belong to diverse morphological classes, including bipolar, bitufted, neurogliaform, and Martinotti cells (Kawaguchi and Kubota, 1997; Silberberg et al., 2005; Jiang et al., 2015; Tremblay et al., 2016). An overall potentiating effect of heterosynaptic plasticity may help non-FS neurons to preserve very weak synapses (with high PPR, low release probability) from further weakening and eventual elimination because of Hebbiantype plasticity. A decrease in PPR associated with potentiation shifts the bulk of response toward earlier spikes in a train (Markram and Tsodyks, 1996), leading to earlier activation of inhibitory neurons during episodes of excessive activity. Net potentiation after such episodes by the mechanism of heterosynaptic plasticity will help to engage non-FS cells (e.g., Martinotti cells controlling vertical spread of activity in a column; Bacci et al., 2005; Adesnik and Scanziani, 2010; Cardin, 2018) earlier, and thus more effectively counteract overexcitation of the column. Because many non-FS cells contact other inhibitory neurons (Jiang et al., 2015), their activation may have complex effects on inhibition and activity in the column.

\section{Outlook}

Differential net effects of heterosynaptic plasticity in FS and non-FS neurons add a new level of complexity in regulation of cortical inhibition. We propose that in FS neurons, weightdependent heterosynaptic plasticity can play a role of homeostatic brakes preventing extreme changes at excitatory inputs, similar to its role in excitatory neurons. In non-FS neurons, heterosynaptic plasticity may help to maintain operation of inhibitory system in a different way: preventing low-probability synapses from elimination by Hebbian-type plasticity, and thus keeping functional inhibitory neurons activated by such synapses. Because low-probability synapses tend to be facilitatory (Markram et al., 1998; Lu et al., 2007) such neurons may serve as brakes activated by excessive firing in the network.

The present results expose a number of questions requiring further research. What are the specific mechanisms of induction and expression of weight-dependent heterosynaptic plasticity in different types of inhibitory cells? Which retrograde signaling systems are mediating presynaptic components of heterosynaptic changes? Primary candidates here are cannabinoid and metabotropic receptors (Bacci et al., 2005; Sarihi et al., 2008) and NOsystem. Indeed, our prior work demonstrated that NO-system is involved in retrograde signaling during induction of heterosynaptic plasticity in pyramidal neurons (Volgushev et al., 2000), and inhibitory neurons are known to express NO-synthase (Estrada and DeFelipe, 1998; Kubota et al., 2011). Here we show that heterosynaptic changes accompany the induction of homosynaptic plasticity; however, how these forms of plasticity interact, and what mechanisms mediate their interaction in cortical interneurons remains to be clarified. Ultimately, understanding how diverse forms of plasticity in specific classes of inhibitory neurons shape diverse inhibitory systems serving distinct functional roles in neuronal networks will be a necessary step toward understanding normal function and causes for dysfunction of cortical inhibition.

\section{References}

Abraham WC, Bear MF (1996) Metaplasticity: the plasticity of synaptic plasticity. Trends Neurosci 19:126-130. 
Abraham WC, Logan B, Wolff A, Benuskova L (2007) "Heterosynaptic" LTD in the dentate gyrus of anesthetized rat requires homosynaptic activity. J Neurophysiol 98:1048-1051.

Adesnik H, Scanziani M (2010) Lateral competition for cortical space by layer-specific horizontal circuits. Nature 464:1155-1160.

Allen K, Monyer H (2015) Interneuron control of hippocampal oscillations. Curr Opin Neurobiol 31:81-87.

Ascoli GA, Alonso-Nanclares L, Anderson SA, Barrionuevo G, BenavidesPiccione R, Burkhalter A, Buzsáki G, Cauli B, Defelipe J, Fairén A, Feldmeyer D, Fishell G, Fregnac Y, Freund TF, Gardner D, Gardner EP, Goldberg JH, Helmstaedter M, Hestrin S, Karube F, et al. (2008) Petilla terminology: nomenclature of features of GABAergic interneurons of the cerebral cortex. Nat Rev Neurosci 9:557-568.

Bacci A, Huguenard JR, Prince DA (2005) Modulation of neocortical interneurons: extrinsic influences and exercises in self-control. Trends Neurosci 28:602-610.

Balaban P, Chistiakova M, Malyshev A, Volgushev M (2004) Dependence of calcium influx in neocortical cells on temporal structure of depolarization, number of spikes, and blockade of NMDA receptors. J Neurosci Res 76:481-487.

Bannon NM, Zhang P, Ilin V, Chistiakova M, Volgushev M (2014) Modulation of synaptic transmission by adenosine in layer $2 / 3$ of the rat visual cortex in vitro. Neuroscience 260:171-184.

Bannon NM, Chistiakova M, Chen JY, Bazhenov M, Volgushev M (2017) Adenosine shifts plasticity regimes between associative and homeostatic by modulating heterosynaptic changes. J Neurosci 37:1439-1452.

Baranauskas G, Mukovskiy A, Wolf F, Volgushev M (2010) The determinants of the onset dynamics of action potentials in a computational model. Neuroscience 167:1070-1090.

Barnes SA, Pinto-Duarte A, Kappe A, Zembrzycki A, Metzler A, Mukamel EA, Lucero J, Wang X, Sejnowski TJ, Markou A, Behrens MM (2015) Disruption of mGluR5 in parvalbumin-positive interneurons induces core features of neurodevelopmental disorders. Mol Psychiatry 20:1161-1172.

Battaglia D, Karagiannis A, Gallopin T, Gutch HW, Cauli B (2013) Beyond the frontiers of neuronal types. Front Neural Circuits 7:13.

Bi GQ, Poo MM (1998) Synaptic modifications in cultured hippocampal neurons: dependence on spike timing, synaptic strength, and postsynaptic cell type. J Neurosci 18:10464-10472.

Buzás P, Eysel UT, Kisvárday ZF (1998) Functional topography of single cortical cells: an intracellular approach combined with optical imaging. Brain Res Brain Res Protoc 3:199-208.

Cardin JA (2018) Inhibitory interneurons regulate temporal precision and correlations in cortical circuits. Trends Neurosci 41:689-700.

Cardin JA, Carlén M, Meletis K, Knoblich U, Zhang F, Deisseroth K, Tsai LH, Moore CI (2009) Driving fast-spiking cells induces gamma rhythm and controls sensory responses. Nature 459:663-667.

Chen HX, Jiang M, Akakin D, Roper SN (2009) Long-term potentiation of excitatory synapses on neocortical somatostatin-expressing interneurons. J Neurophysiol 102:3251-3259.

Chen JY, Lonjers P, Lee C, Chistiakova M, Volgushev M, Bazhenov M (2013) Heterosynaptic plasticity prevents runaway synaptic dynamics. J Neurosci 33:15915-15929.

Chen SX, Kim AN, Peters AJ, Komiyama T (2015) Subtype-specific plasticity of inhibitory circuits in motor cortex during motor learning. Nat Neurosci 18:1109-1115.

Chistiakova M, Bannon NM, Bazhenov M, Volgushev M (2014) Heterosynaptic plasticity: multiple mechanisms and multiple roles. The Neuroscientist 20:483-498.

Chistiakova M, Bannon NM, Chen JY, Bazhenov M, Volgushev M (2015) Homeostatic role of heterosynaptic plasticity: models and experiments. Front Comput Neurosci 9:89.

Cowan AI, Stricker C, Reece LJ, Redman SJ (1998) Long-term plasticity at excitatory synapses on aspinous interneurons in area CAl lacks synaptic specificity. J Neurophysiol 79:13-20.

Debanne D, Gähwiler BH, Thompson SM (1999) Heterogeneity of synaptic plasticity at unitary CA1-CA3 and CA3-CA3 connections in rat hippocampal slice cultures. J Neurosci 19:10664-10671.

DeFelipe J, López-Cruz PL, Benavides-Piccione R, Bielza C, Larrañaga P, Anderson S, Burkhalter A, Cauli B, Fairén A, Feldmeyer D, Fishell G, Fitzpatrick D, Freund TF, González-Burgos G, Hestrin S, Hill S, Hof PR, Huang J, Jones EG, Kawaguchi Y, et al. (2013) New insights into the classification and nomenclature of cortical GABAergic interneurons. Nat Rev Neurosci 14:202-216.

Dorrn AL, Yuan K, Barker AJ, Schreiner CE, Froemke RC (2010) Developmental sensory experience balances cortical excitation and inhibition. Nature 465:932-936.

Druckmann S, Hill S, Schürmann F, Markram H, Segev I (2013) A hierarchical structure of cortical interneuron electrical diversity revealed by automated statistical analysis. Cereb Cortex 23:2994-3006.

Estrada C, DeFelipe J (1998) Nitric oxide-producing neurons in the neocortex: morphological and functional relationship with intraparenchymal microvasculature. Cereb Cortex 8:193-203.

Feldman DE (2009) Synaptic mechanisms for plasticity in neocortex. Annu Rev Neurosci 32:33-55.

Freund TF, Martin KA, Soltesz I, Somogyi P, Whitteridge D (1989) Arborisation pattern and postsynaptic targets of physiologically identified thalamocortical afferents in striate cortex of the macaque monkey. J Comp Neurol 289:315-336.

Froemke RC, Merzenich MM, Schreiner CE (2007) A synaptic memory trace for cortical receptive field plasticity. Nature 450:425-429.

Gandhi SP, Yanagawa Y, Stryker MP (2008) Delayed plasticity of inhibitory neurons in developing visual cortex. Proc Natl Acad Sci USA 105:16797-16802.

Garkun Y, Maffei A (2014) Cannabinoid-dependent potentiation of inhibition at eye opening in mouse V1. Front Cell Neurosci 8:46.

Gentet LJ (2012) Functional diversity of supragranular GABAergic neurons in the barrel cortex. Front Neural Circuits 6:52.

Hensch TK (2005) Critical period plasticity in local cortical circuits. Nat Rev Neurosci 6:877-888.

Hensch TK, Fagiolini M, Mataga N, Stryker MP, Baekkeskov S, Kash SF (1998) Local GABA circuit control of experience-dependent plasticity in developing visual cortex. Science 282:1504-1508.

$\mathrm{Hu} \mathrm{H}$, Gan J, Jonas P (2014) Interneurons. Fast-spiking, parvalbumin ${ }^{+}$ GABAergic interneurons: from cellular design to microcircuit function. Science 345:1255263.

Huang S, Huganir RL, Kirkwood A (2013) Adrenergic gating of Hebbian spike-timing-dependent plasticity in cortical interneurons. J Neurosci 33: 13171-13178.

Jiang X, Shen S, Cadwell CR, Berens P, Sinz F, Ecker AS, Patel S, Tolias AS (2015) Principles of connectivity among morphologically defined cell types in adult neocortex. Science 350:aac9462.

Kawaguchi Y, Kubota Y (1997) GABAergic cell subtypes and their synaptic connections in rat frontal cortex. Cereb Cortex 7:476-486.

Kubota Y, Shigematsu N, Karube F, Sekigawa A, Kato S, Yamaguchi N, Hirai Y, Morishima M, Kawaguchi Y (2011) Selective coexpression of multiple chemical markers defines discrete populations of neocortical GABAergic neurons. Cereb Cortex 21:1803-1817.

Lamsa K, Heeroma JH, Kullmann DM (2005) Hebbian LTP in feed-forward inhibitory interneurons and the temporal fidelity of input discrimination. Nat Neurosci 8:916-924.

Lamsa KP, Heeroma JH, Somogyi P, Rusakov DA, Kullmann DM (2007) Anti-Hebbian long-term potentiation in the hippocampal feedback inhibitory circuit. Science 315:1262-1266.

Larsen RS, Sjöström PJ (2015) Synapse-type-specific plasticity in local circuits. Curr Opin Neurobiol 35:127-135.

Lee CM, Stoelzel C, Chistiakova M, Volgushev M (2012) Heterosynaptic plasticity induced by intracellular tetanization in layer $2 / 3$ pyramidal neurons in rat auditory cortex. J Physiol 590:2253-2271.

Lefort S, Gray AC, Turrigiano GG (2013) Long-term inhibitory plasticity in visual cortical inhibitory layer 4 switches sign at the opening of the critical period. Proc Natl Acad Sci U S A 110:E4540-E4547.

Liguz-Lecznar M, Urban-Ciecko J, Kossut M (2016) Somatostatin and somatostatin-containing neurons in shaping neuronal activity and plasticity. Front Neural Circuits 10:48.

Lu JT, Li CY, Zhao JP, Poo MM, Zhang XH (2007) Spike-timing-dependent plasticity of neocortical excitatory synapses on inhibitory interneurons depends on target cell type. J Neurosci 27:9711-9720.

Lynch GS, Dunwiddie T, Gribkoff V (1977) Heterosynaptic depression: a postsynaptic correlate of long-term potentiation. Nature 266:737-739.

Maffei A, Nelson SB, Turrigiano GG (2004) Selective reconfiguration of layer 4 visual cortical circuitry by visual deprivation. Nat Neurosci 7:1353-1359. 
Maffei A, Nataraj K, Nelson SB, Turrigiano GG (2006) Potentiation of cortical inhibition by visual deprivation. Nature 443:81-84.

Markram H, Tsodyks M (1996) Redistribution of synaptic efficacy between neocortical pyramidal neurons. Nature 382:807-810.

Markram H, Wang Y, Tsodyks M (1998) Differential signaling via the same axon of neocortical pyramidal neurons. Proc Natl Acad Sci U S A 95:5323-5328.

Markram H, Toledo-Rodriguez M, Wang Y, Gupta A, Silberberg G, Wu C (2004) Interneurons of the neocortical inhibitory system. Nat Rev Neurosci 5:793-807.

Miska NJ, Richter LM, Cary BA, Gjorgjieva J, Turrigiano GG (2018) Sensory experience inversely regulates feedforward and feedback excitationinhibition ratio in rodent visual cortex. eLife 7:e38846.

Monier C, Chavane F, Baudot P, Graham LJ, Frégnac Y (2003) Orientation and direction selectivity of synaptic inputs in visual cortical neurons: a diversity of combinations produces spike tuning. Neuron 37:663-680.

Moore AK, Weible AP, Balmer TS, Trussell LO, Wehr M (2018) Rapid rebalancing of excitation and inhibition by cortical circuitry. Neuron 97:1341-1355.e6.

Murthy VN, Sejnowski TJ, Stevens CF (1997) Heterogeneous release properties of visualized individual hippocampal synapses. Neuron 18:599-612.

Naundorf B, Wolf F, Volgushev M (2006) Unique features of action potential initiation in cortical neurons. Nature 440:1060-1063.

Okun M, Lampl I (2008) Instantaneous correlation of excitation and inhibition during ongoing and sensory-evoked activities. Nat Neurosci 11: $535-537$.

Oleskevich S, Clements J, Walmsley B (2000) Release probability modulates short-term plasticity at a rat giant terminal. J Physiol 524:513-523.

Ozeki H, Finn IM, Schaffer ES, Miller KD, Ferster D (2009) Inhibitory stabilization of the cortical network underlies visual surround suppression. Neuron 62:578-592.

Sarihi A, Jiang B, Komaki A, Sohya K, Yanagawa Y, Tsumoto T (2008)
Metabotropic glutamate receptor type 5-dependent long-term potentiation of excitatory synapses on fast-spiking GABAergic neurons in mouse visual cortex. J Neurosci 28:1224-1235.

Silberberg G, Grillner S, LeBeau FE, Maex R, Markram H (2005) Synaptic pathways in neural microcircuits. Trends Neurosci 28:541-551.

Sohal VS, Zhang F, Yizhar O, Deisseroth K (2009) Parvalbumin neurons and gamma rhythms enhance cortical circuit performance. Nature 459:698-702.

Tremblay R, Lee S, Rudy B (2016) GABAergic interneurons in the neocortex: from cellular properties to circuits. Neuron 91:260-292.

Tsodyks MV, Markram H (1997) The neural code between neocortical pyramidal neurons depends on neurotransmitter release probability. Proc Natl Acad Sci U S A 94:719-723.

Vidyasagar TR, Pei X, Volgushev M (1996) Multiple mechanisms underlying the orientation selectivity of visual cortical neurones. Trends Neurosci 19:272-277.

Volgushev M, Voronin LL, Chistiakova M, Singer W (1997) Relations between long-term synaptic modifications and paired-pulse interactions in the rat neocortex. Eur J Neurosci 9:1656-1665.

Volgushev M, Balaban P, Chistiakova M, Eysel UT (2000) Retrograde signaling with nitric oxide at neocortical synapses. Eur J Neurosci 12: 4255-4267.

Volgushev M, Chen JY, Ilin V, Goz R, Chistiakova M, Bazhenov M (2016) Partial breakdown of input specificity of STDP at individual synapses promotes new learning. J Neurosci 36:8842-8855.

Voronin LL (1993) On the quantal analysis of hippocampal long-term potentiation and related phenomena of synaptic plasticity. Neuroscience 56:275-304.

Wehr M, Zador AM (2003) Balanced inhibition underlies tuning and sharpens spike timing in auditory cortex. Nature 426:442-446.

Zhang P, Bannon NM, Ilin V, Volgushev M, Chistiakova M (2015) Adenosine effects on inhibitory synaptic transmission and excitation-inhibition balance in the rat neocortex. J Physiol 593:825-841. 